\title{
Pattern selection when a layer buckles on a soft substrate
}

\author{
Nontawit Cheewaruangroj ${ }^{1}$ and John S. Biggins ${ }^{1,2}$ \\ ${ }^{1}$ Cavendish Laboratory, University of Cambridge, \\ 19 JJ Thomson Avenue, Cambridge CB3 OHE, United Kingdom \\ ${ }^{2}$ Engineering Department, University of Cambridge, \\ Trumpington Street, Cambridge CB2 1PZ, United Kingdom
}

(Dated: April 28, 2019)

\begin{abstract}
If a neo-Hookean elastic layer adhered to a neo-Hookean substrate grows equibiaxially, it will buckle into a pattern of topography. Here, we combine higher order perturbation theory and finite element numerics to predict the pattern formed just beyond the buckling threshold. More precisely, we construct series solutions corresponding to hexagonal, square and stripe patterns, and expand the elastic energy for each pattern as a Landau-like energy series in the topography amplitude. We see that, for square and stripe patterns, the elastic energy is invariant under topography inversion, making the instabilities supercritical. However, since patterns of hexagonal dents are physically different to patterns of hexagonal bumps, the hexagonal energy lacks this invariance. This lack introduces a cubic term which causes hexagonal patterns to be formed subcritically and hence energetically favoured. Our analytic calculation of the cubic term allows us to determine that dents are favoured in incompressible systems, but bumps are favored in sufficiently compressible systems. Finally, we consider a stiff layer sandwiched between an identical pair of substrate and superstrate. This system has topography inversion symmetry, so hexagons form supercritically, and square patterns are favoured. We use finite elements to verify our theoretical predictions for each pattern, and confirm which pattern is selected. Previous work has used a simplified elastic model (plate \& linear elastic substrate) that possesses invariance under topography inversion, and hence incorrectly predicted square patterns. Our work demonstrates that large strain geometry is sufficient to break this symmetry and explain the hexagonal dent patterns observed in buckling experiments.
\end{abstract}

\section{INTRODUCTION}

If a thin elastic layer is adhered to a compliment substrate and then caused to enter compression, it will buckle into a pattern of relief [1]. Such layer/substrate buckling is a rich pattern-forming system, with a zoo of different topographies emerging depending on the layer/substrate stiffness ratio, the layer/substrate thickness ratio, the degree of compressibility, and the magnitude and degree of anisotropy of compression. This richness has been exploited by evolution, which uses layer/substrate buckling to sculpt organs during development [2-9], including the folds on the surface of the mammalian brain [10-16], and the loops and villi of the gut [17-19]. Layer/substrate buckling also offers an attractive mechanism for scientists and engineers to generate and switch topography, providing a route to robust shape generation [20,21] and allowing the creation of surfaces with switchable adhesion [22, 23], wetability [24-26], photonics [27-29] and encapsulation [30, 31].

On a theoretical level, there has been much work on characterizing the thresholds and wavelengths for these buckling instabilities [1, 32-39], but less is understood about the emergent patterns. In this paper, we use higher order perturbation theory to tackle the problem of pattern selection in an important subset of such buckling instabilities, which occur when the elastic layer enters compression due to isotropic (equibiaxial) growth/swelling/expansion relative to the substrate. Our main conclusion is that, close to threshold, such layers buckle subcritically into patterns of hexagonal dents.

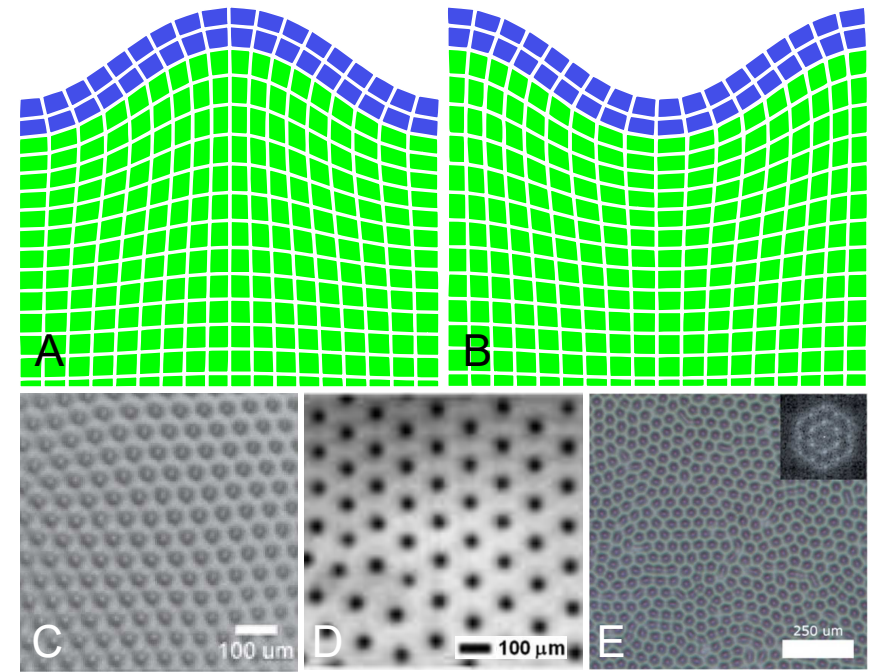

FIG. 1. (A and B) Schematic diagrams showing deformation of a bump and a dent. (C-E) Optical microsopy images of hexagonal dents formed by solvent swelling a stiff layer on a compliant soft substrate of hydrogels, taken from [40-42].

Although our calculations are for specific layer/substrate properties (neo-Hookean materials, infinite substrate), we argue that hexagonal patterns are a consequence of the symmetry of the system, and hence will be generic in all transversely isotropic elastic surface instabilities. Our layer/substrate system spans three distinct regimes. The best studied is a stiff layer on a soft substrate, such as a metal layer on an elastomer substrate. If such a system 
is heated, the metal will expand relative to the substrate, enter compression, and then buckle into a pattern of relief. This process is a form of Euler buckling, but with a finite wavelength emerging from a trade-off between the bending stiffness of the layer and the substrate elasticity $[1,43]$. Experimentally, such systems produce herringbone patterns far beyond threshold [40, 44], which have been modelled theoretically using an elastic (Fopplvon Karman) plate adhered to a linear elastic substrate [42, 44, 45]. However, close to threshold, such models predict square/checkerboard buckling patterns [42, 45], whereas patterns of hexagonal dents are observed experimentally and numerically (Figs 1C-E) [40-42, 46, 47]. Here, we argue that the plate/linear-substrate model fails because it possesses an unphysical inversion symmetry. A normal displacement of the plate $u_{z}(x, y)$ encoding a bump (Fig. 1A) is physically different from $-u_{z}(x, y)$ encoding a dent (Fig. 1B), and this difference must underpin the experimental formation of hexagonal dents rather than bumps. In contrast, the plate/linear-substrate energy is invariant under such topography inversion as the plate itself is up/down symmetric, and the (linear-elastic) substrate energy is a quadratic function of displacement. A theoretical prediction of the hexagonal dent pattern clearly requires a model without this inversion symmetry.

The inspiration for how to proceed comes from the other two regimes. The opposite regime is a soft layer on a rigid substrate, for example a hydrogel layer adhered to a glass slide. If the hydrogel is induced to swell, it enters compression, and undergoes a surface instability known as creasing or sulcification [48-51], which forms a pattern of cusped self-contacting surface furrows. To understand this instability theoretically, one must use a geometrically nonlinear hyperelastic model for the soft layer (typically a neo-Hookean model) with a clamped bottom boundary modelling the substrate [52]. Such a model dramatically breaks topographical inversion symmetry via the non-linearities of large deformation geometry: simply inverting a cusped surface furrow (a sulcus/crease) would yield an unphysical state with singular energy. Previous authors have studied pattern formation in this system using higher order perturbation theory [53, 54] and found that patterns of hexagonal dents are indeed favored. However, ironically, experimental [55] and numerical [56] studies show that in this regime, the pattern near threshold is in fact a checkerboard of cusped furrows. The mismatch arises because sulcification occurs via an exotic non-linear instability [50] which cannot be treated perturbatively, but these theoretical studies nevertheless establish that geometrically non-linear elasticity can break inversion symmetry and favors hexagonal dents.

Between these extremes is an intermediate regime: a growing soft-layer adhered to a soft substrate. This mechanically intriguing regime spans the transition from conventional stiff-soft wrinkling to soft-stiff sulcification, and characterizes many biological buckling systems [2-
19]. Recently there has been much work on the onset of instability in this regime, using geometrically nonlinear neo-Hookean elastic models for both the layer and the substrate [47, 57-59]. These studies have largely focused on 2-D linear stability analysis (i.e. uniaxial growth/compression yielding pure sinusoidal stripe patterns) and have documented how the wavelength and threshold of the instability move as a function of layer/substrate modulus ratio, and identified the modulus ratio at which conventional finite wavelength buckling is replaced by sulcification. What emerges clearly from these studies is that, at least in this intermediate regime, geometric non-linearity plays a role in even the onset of instability, with the wavelengths and thresholds deviating from those predicted by the traditional plate/linearsubstrate model. Further evidence of the role of substrate non-linearity is provided in [60-62] where a substrate pre-stretch/pre-compression is shown to favor the formation of surface ridges/furrows respectively, demonstrating that substrate non-linearity not only matters, but also breaks pattern inversion symmetry.

In this paper, we pursue the natural next step by taking such a fully geometrically non-linear elastic model of a neo-Hookean growing layer on a neo-Hookean substrate, and using it to address pattern selection. This fully non-linear model correctly lacks topography inversion symmetry. In the first section of our paper, we argue that, on symmetry grounds, this means hexagonal buckling patterns will generically appear subcritically and be favored near threshold. In the main body of the paper, we verify and extend this basic understanding by explicitly conducting higher order perturbation theory on the neo-Hookean layer/substrate, to evaluate the amplitude and energy of stripe, square and hexagonal elastic solutions near threshold. The full neo-Hookean model is significantly more complicated than the previously used plate/linear-substrate and neo-Hookean-layer/clampedbottom-boundary models, so we are only able to evaluate the higher order fields using computer algebra. However, at each stage, we are able to verify our computeralgebra predictions against symmetry-constrained finite element calculations, lending confidence to their accuracy. These analytic and computational calculations reveal that, for incompressible systems, hexagonal dent patterns are favored, whereas for sponge-like compressible systems, hexagonal bumps are favored. These conclusions break down when the layer is less than twice as stiff as the substrate, as then all types of patterns become subcritical, an expected signature of the transition to the sulcification regime. Finally, to emphasize the relationship between hexagonal patterns and inversion symmetry, we consider a stiff layer sandwiched between a matching pair of soft substrate and superstrate. This system has a true inversion symmetry, and correspondingly forms supercritical square patterns rather than subcritical hexagons. 


\section{GEOMETRICALLY NON-LINEAR MODEL FOR LAYER/SUBSTRATE}

Our system consists of a compressed layer adhered to a relaxed infinite substrate, and we seek to determine the selected buckling pattern by finding the configuration that minimizes the system's total elastic energy. More precisely, in Cartesian coordinates, we consider an elastic half space occupying $z<0$ coated with an elastic layer occupying $0<z<a$. In this flat configuration, the substrate is elastically relaxed, but the layer has undergone equibiaxial growth/swelling/expansion post adhesion to the substrate such that, if it were released, it would extend equibiaxially in the $x-y$ plane by a factor of $g$. When $g$ exceeds a threshold value $g^{*}$, the layer will buckle into a non-flat pattern of topography with lower elastic energy. If, in this buckling, the layer/substrate undergo a displacement field $\mathbf{u}=\left(u_{x}(x, y, z), u_{y}(x, y, z), u_{z}(x, y, z)\right)$, the full local shape changes are encoded by the deformation gradient (also known as the transformation matrix) $F_{i j}=\delta_{i j}+\partial_{j} u_{i}$ (where $\delta_{i j}$ is the Kronecker delta symbol), and the local elastic energy density is a function of this shape change $W(F)$. The total elastic energy is then the integral of $W(F)$ over the entire $-\infty<z<a$ volume, but since this energy is extensive, we normalize per-unit-area in the $x-y$ plane,

$$
E_{e l}=\int_{z=-\infty}^{z=a}\langle W(F)\rangle \mathrm{d} z .
$$

where angle brackets denote and in-plane average, $\langle f\rangle=$ $\lim _{l \rightarrow \infty} \frac{1}{l^{2}} \int_{x=-l}^{x=l} \int_{y=-l}^{y=l} f \mathrm{~d} x \mathrm{~d} y$. Minimizing this energy with respect to variations in $\mathbf{u}$ leads to the expected bulk equation of mechanical equilibrium

$$
\nabla \cdot \sigma=\mathbf{0}
$$

where $\sigma=\frac{\partial W}{\partial \nabla \mathbf{u}}$ is the large-strain "first Piola-Kirchhoff" (PK1) stress tensor, a two-point tensor relating areas in the reference state to forces in the deformed state. This bulk equation is augmented by the natural boundary conditions, which require the top surface to be stress free, and the stress to be continuous at the layer/substrate interface:

$$
\left.\sigma \cdot \hat{\mathbf{z}}\right|_{z=a}=\mathbf{0} \quad \text { and }\left.\quad \sigma \cdot \hat{\mathbf{z}}\right|_{z=0^{+}}=\left.\sigma \cdot \hat{\mathbf{z}}\right|_{z=0^{-}} \cdot
$$

In addition, layer/substrate adhesion requires the displacement field to be continuous at the interface

$$
\left.\mathbf{u}\right|_{z=0^{+}}=\left.\mathbf{u}\right|_{z=0^{-}} .
$$

For most of our work, we model the substrate and layer as incompressible neo-Hookean solids with shear moduli $\mu_{2}$ and $\mu_{1} \equiv \eta \mu_{2}$. Renormalizing both moduli by $\mu_{2}$, the modulus and growth in both the layer and substrate are given by the piecewise functions:

$$
(\tilde{\mu}, \tilde{g})= \begin{cases}(\eta, g) & 0<z<a \\ (1,1) & z<0\end{cases}
$$

Incompressibility requires $\operatorname{Det}(F)=1$, meaning that, if material from either the layer or substrate was allowed to relax to its elastic ground-state, it would undergo the homogeneous deformation

$$
G=\left(\begin{array}{ccc}
\tilde{g} & 0 & 0 \\
0 & \tilde{g} & 0 \\
0 & 0 & 1 / \tilde{g}^{2}
\end{array}\right)
$$

which is identity in the substrate. The full neo-Hookean form for $W(F)$, covering both the layer and the substrate, is thus

$$
W=\frac{1}{2} \tilde{\mu}\left[\operatorname{Tr}\left(F \cdot G^{-1} \cdot G^{-T} \cdot F^{T}\right)-3\right]-P(\operatorname{Det}(F)-1),
$$

where $P$ is a Lagrange multiplier pressure field enforcing incompressibility. In this case, the PK1 stress tensor evaluates to

$$
\sigma=\tilde{\mu} F G^{-1} G^{-T}-P \operatorname{cof}(F),
$$

where $\operatorname{cof}(F)$ refers to the cofactor of $F$, and one must also minimize the energy over variations in $P$ yielding a further bulk equation of incompressibility:

$$
\operatorname{Det}(F)=1 \text {. }
$$

\section{APPROACH: SERIES EXPANSION OF THE ENERGY IN THE AMPLITUDE}

Our task is now to construct solutions to the above bulk/boundary equations for different types of patterns and evaluate their corresponding elastic energies. In general, such patterns will have large amplitudes, and require intractable fully non-linear solutions, so our strategy is to look at patterns that are perturbatively close to the flat state. To do this, we conduct the elastic energy minimization in two stages. We first minimize the energy over configurations with small amplitude $\epsilon$ then, second, minimize the energy over $\epsilon$ to find the true minimum. This two-step approach has two advantages: it circumvents difficulties of self-consistency and solvability that commonly complicate higher order perturbation theory, and clarifies the origin hexagonal patterns. To constrain the amplitude during the first stage, we introduce a Lagrange multiplier $L$ which enforces the root-mean-square amplitude of the surface:

$$
E=E_{e l}+L\left\langle\left. u_{z}^{2}\right|_{z=a}-\epsilon^{2}\right\rangle .
$$

The new term only contributes on the top $(z=a)$ surface, so minimizing $E$ with respect to variations in $\mathbf{u}, L$ (and $P$ for the incompressible case) yields the same bulk and boundary equations as before, except it introduces an external stress at the top surface $2 L u_{z}(x, y, z=a) \hat{\mathbf{z}}$, so the boundary condition on the top surface is modified to:

$$
\left.\left(\sigma \cdot \hat{\mathbf{z}}+2 L u_{z} \hat{\mathbf{z}}\right)\right|_{z=a}=\mathbf{0} \text {. }
$$


This is equivalent to the solvability conditions that need to be imposed at the higher order post buckling analysis [63]. Finally, minimizing $E$ over $L$ yields the imposed constraint on the amplitude,

$$
\sqrt{\left.\left\langle u_{z}^{2}\right\rangle\right|_{z=a}}=|\epsilon| .
$$

Since $\epsilon$ is now guaranteed to be a small amplitude, we can expand all the fields in a series expansion in $\epsilon$ :

$$
\begin{aligned}
\mathbf{u}(x, y, z) & =\sum_{n=1}^{\infty} \epsilon^{n} \mathbf{u}_{n}(x, y, z) \\
P(x, y, z) & =\tilde{\mu} P_{0}(z)+\sum_{n=1}^{\infty} \epsilon^{n} \tilde{\mu} P_{n}(x, y, z) \\
L & =L_{0}+\sum_{n=1}^{\infty} \epsilon^{n} L_{n} .
\end{aligned}
$$

Our strategy is then to substitute these expansions into the bulk and boundary equations, expand the equations in $\epsilon$, and solve order-by-order to build up series solutions for the fields. These solutions can then be substituted into $E$, to yield a Landau-like series solution of the energy in $\epsilon$ :

$$
E=E_{0}+\sum_{n=1}^{\infty} \epsilon^{n} E_{n}
$$

Finally, we conduct the second step: we truncate this energy series at sufficient order, and minimize over $\epsilon$ to find the amplitude and elastic energy of the solution.

\section{THE INEVITABILITY OF HEXAGONAL PATTERNS}

In the above scheme, the first order equations $(\mathcal{O}(\epsilon))$ will be linear equations in the first order fields, corresponding to the equations of linear stability analysis. Since we expect periodic buckling patterns, it is natural to express the first order fields as a sum of Fourier components with $k$-vectors in the $x-y$ plane. However, since the first order equations are linear, these components can be treated separately, so it is sufficient to consider a single Fourier component such as $u_{1 z}=f_{1}(z) \cos (k x)$, which would give a stripe buckling pattern on the surface.

Having made this initial ansatz, the full set of first order fields can be found from the first order bulk and boundary equations. Upon then evaluating the energy series, one inevitably will find that $E_{1}$ vanishes as the initial flat state is always an equilibrium and hence a stationary point in the energy landscape, so its first order energy variation vanishes for all perturbations. Similarly, the first order solutions are sufficient to evaluate $E_{2}$, and the sign of $E_{2}$ then determines whether, for very small amplitudes, the buckling solution saves or costs energy, and hence whether the flat state is stable. By definition, the transition from stable to unstable happens at $g^{*}$, so we expect the energy series to take the Landau-like form, $E=E_{0}+C_{2}\left(g-g^{*}\right) \epsilon^{2}$, where $C_{2}<0$. In general, the value of $g^{*}$ that emerges from this calculation will depend on the details of the model (modulus ratio, compressible vs incompressible etc.) and the value of $k$. It is then necessary to find the $k$ with the smallest $g^{*}$, which will be the first unstable buckling mode. The first order solution (linear stability analysis) thus delivers the threshold $g^{*}$ and wavelength $\lambda=2 \pi / k$ of the buckling instability.

However, linear stability analysis is insufficient to predict the stripe amplitude, as one cannot minimize $E$ over $\epsilon$ without higher order terms. Furthermore, since all inplane directions are equivalent, the values of $g^{*}$ and $C_{2}$ do not depend on the (in-plane) direction of the $k$ vector, so linear stability analysis cannot predict the direction stripes form in. Indeed, any linear combination of stripes in different directions but with the same magnitude of $k$ must also solve the linear first order equations and become unstable at the same threshold, but will produce very different patterns of topography. In this paper, we consider three basic topographical patterns, stripes, squares (checkerboards) and hexagons, encoded by the following linear combinations:

$$
\begin{aligned}
\text { stripe : } & u_{1 z} \sim \cos (k x) \\
\text { square : } & u_{1 z} \sim \cos (k x)+\cos (k y) \\
\text { hexagon: } & u_{1 z} \sim \cos (k x)+\cos \left(k x_{1}\right)+\cos \left(k x_{2}\right),
\end{aligned}
$$

where $x_{1}=\frac{x}{2}+\frac{\sqrt{3} y}{2}$ and $x_{2}=-\frac{x}{2}+\frac{\sqrt{3} y}{2}$. Since these patterns will all have the same threshold under linear stability analysis, to predict which pattern forms one must go beyond linear stability analysis, and calculate higher order elastic fields and higher order terms in $E$ to establish which pattern is the true energetic minimizer. We note that herringbone patterns, which have different wavelengths in the $x$ and $y$ directions, are guaranteed to have a higher threshold, so they are not candidates for the energy minimizer near threshold, and must arise in stiff layer buckling via a secondary bifurcation.

Although these different patterns generate first order fields that are trivial linear combinations of the initial stripe solution, these different starting points generate different higher order fields, and different higher order terms in the energy. In general, it is sufficient to solve the fields to third order, which allows us to evaluate the energy series to fourth order:

$$
E=E_{0}+C_{2}\left(g-g^{*}\right) \epsilon^{2}+C_{3} \epsilon^{3}+C_{4} \epsilon^{4}+\mathcal{O}\left(\epsilon^{5}\right),
$$

where the values of $C_{3}$ and $C_{4}$ will depend on the pattern in question. The key observation is that, as clearly seen in Fig. 2, inverting the first order stripe and square patterns (i.e. setting $\epsilon \rightarrow-\epsilon$ ) is equivalent to a simple translation of the pattern in the $x-y$ plane, and therefore cannot change the energy of the solution. In contrast, with hexagons the same inversion turns a pattern of bumps into a non-equivalent pattern of dents that should have a 
A

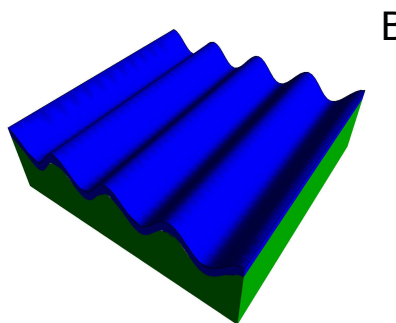

C

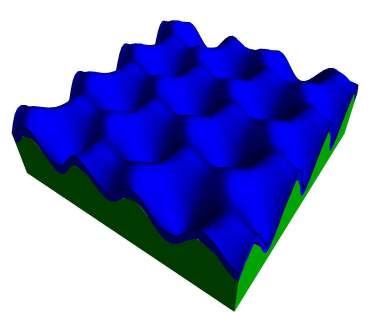

B

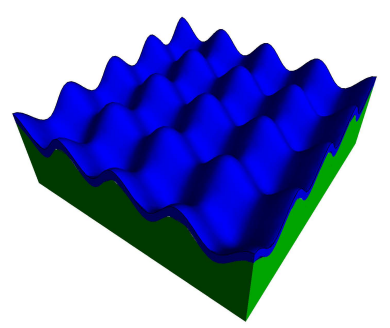

D

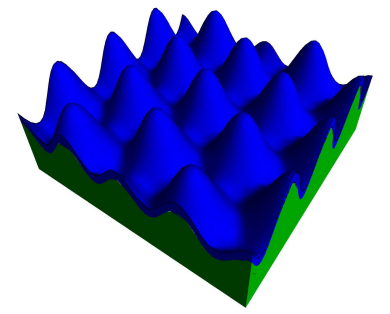

FIG. 2. Schematic diagram for different patterns: (A) stripe, (B) square, (C) positive hexagon and (D) negative hexagon.

different elastic energy. This inversion symmetry of the first order stripe and square patterns requires the coefficient of the odd orders to vanish since $+\epsilon$ and $-\epsilon$ are equivalent. The energy expansion becomes

$$
E=E_{0}+C_{2}\left(g-g^{*}\right) \epsilon^{2}+C_{4} \epsilon^{4}+\mathcal{O}\left(\epsilon^{5}\right) .
$$

If $C_{4}>0$, then, for small amplitude, one can truncate this energy at $\mathcal{O}\left(\epsilon^{4}\right)$ and minimize over $\epsilon$ to find the minimum energy amplitude

$$
\epsilon^{*}= \begin{cases}\sqrt{\frac{-C_{2}\left(g-g^{*}\right)}{2 C_{4}}} & g>g^{*}, \\ 0 & g<g^{*} .\end{cases}
$$

The amplitude increases continuously (supercritically) for $g>g^{*}$, so the amplitude is indeed arbitrarily small close to threshold, and the series truncation is self consistent. Past threshold, the energy of the buckled state is

$$
E=E_{0}-\frac{C_{2}^{2}\left(g-g^{*}\right)^{2}}{4 C_{4}}
$$

which falls continuously from $E_{0}$ beyond threshold. Conversely, for the hexagonal patterns, $C_{3}$ will not vanish. This cubic term is always unbounded from below, so one still needs the fourth order term before truncating:

$$
E=E_{0}+C_{2}\left(g-g^{*}\right) \epsilon^{2}+C_{3} \epsilon^{3}+C_{4} \epsilon^{4} .
$$

In this energy, a second minima appears (separate to the one at $\epsilon^{*}=0$ ) for $g>g^{*}+\frac{9 C_{3}^{2}}{32 C_{4} C_{2}}$ at

$$
\epsilon^{*}=-\frac{3 C_{3}}{8 C_{4}}\left(1+\sqrt{1-\frac{32 C_{4} C_{2}\left(g-g^{*}\right)}{9 C_{3}^{2}}}\right) .
$$

In loading (i.e. increasing $g$ ), when $g$ exceeds $g^{*}$, the $\epsilon=0$ minimum becomes a maximum and looses stability,
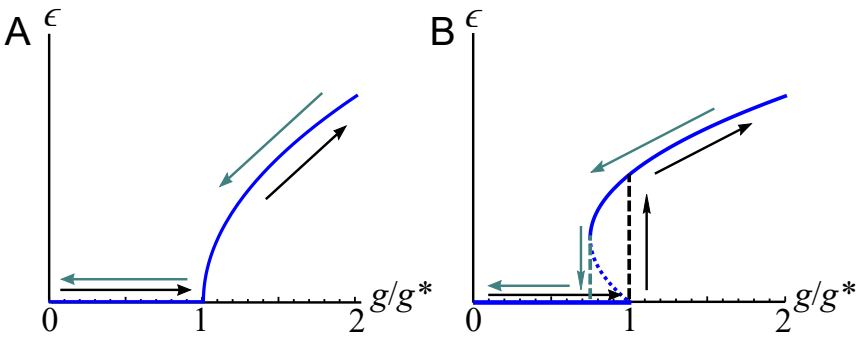

FIG. 3. The loading-unloading curve for (A) supercritical instability and (B) subcritical instability. The black arrow denotes loading, the grey arrow unloading. Dashed line shows the jump of the amplitude at the threshold. Dotted line denotes a branch of an unstable solution.

and the solution will jump to the second minimum which, at threshold, has a finite amplitude of

$$
\epsilon=-\frac{3 C_{3}}{4 C_{4}}
$$

Thus the sign of $C_{3}$ determines the sign of $\epsilon$ and hence whether hexagonal dents or bumps are formed. In unloading, this high-amplitude minimum remains stable until it vanishes when $g=g^{*}+\frac{9 C_{3}^{2}}{32 C_{4} C_{2}}$, at which point the system will jump back to the flat $\epsilon=0$ state. The instability is subcritical (first order, discontinuous) and has a non-trivial hysteresis loop. These two different behaviors are illustrated in Fig. 3. Importantly, at threshold in the hexagonal case, the energy also jumps, to a value of:

$$
E=E_{0}-\frac{27 C_{3}^{4}}{256 C_{4}^{3}}
$$

This finite energy jump at threshold will always place hexagonal patterns below the continuously falling energies generated by square and stripe patterns, so we conclude that hexagonal patterns will be formed. This conclusion is rather general, since the same symmetry considerations will apply to any transversely isotropic surface elastic instability.

We note that the truncation of the energy series is problematic in the hexagonal case, since the instability jumps to a finite amplitude at threshold, so the amplitude $\epsilon$ is not arbitrarily small, and the removed terms may be important. The resulting finite-amplitude hexagonal states may therefore differ appreciably from the actual hexagonal solutions, though in practice we find the disagreements are small. Moreover, since the theory is accurate for small $\epsilon$, it is guaranteed to capture the loss of stability of the flat state correctly, including whether the transition is subcritical, and which sign of $\epsilon$ is chosen, even if it fails to capture the full details high-amplitude solution. Hence, the approach will predict the category of the pattern in spite of the missing details. 


\section{PATTERN FORMATION IN AN INCOMPRESSIBLE NEO-HOOKEAN LAYER AND SUBSTRATE}

Although the above argument offers strong reasons to expect hexagonal buckling patterns, there are still good reasons to explicitly conduct the perturbation theory and compute the energy coefficients. Firstly, it is also possible for the symmetry argument to fail. If $C_{4}$ is negative for squares or stripes then these patterns will also form subcritically, so the theory does not reveal which pattern is preferred. This is because the energy series need to be evaluated to higher orders $\left(\mathcal{O}\left(\epsilon^{6}\right)\right.$ or higher) to make the amplitude bounded from below and there will be a jump in the amplitude at the threshold. In addition, the $C_{3}$ coefficient may vanish "accidentally" in a given system, even though it is permitted on symmetry grounds, making hexagonal patterns also supercritical, in which case the value of $C_{4}$ for the different patterns will determine the optimum pattern. Secondly, even if the argument holds, it is still necessary to compute the coefficients to determine the sign of $C_{3}$, and hence whether patterns of hexagonal bumps or dents will be favoured. In this section, we explicitly compute the fields and energy coefficients for the incompressible neoHookean layer/substrate system described in Section II. We first note that, following the series expansion in eqn. (13), we can also expand $F, \sigma$ and $W$ in $\epsilon$ :

$$
\begin{gathered}
F=I+\sum_{n=1}^{\infty} \epsilon^{n} F_{n} \\
\sigma=\sigma_{0}+\sum_{n=1}^{\infty} \epsilon^{n} \sigma_{n} \\
W=W_{0}+\sum_{n=1}^{\infty} \epsilon^{n} W_{n} .
\end{gathered}
$$

From the definition of $F$, it is immediately clear that $F_{n}=\nabla \mathbf{u}_{n}$, but finding the corresponding expressions for $\sigma_{n}$ and $W_{n}$ is more involved, as they involve $\operatorname{Det}(F)$ and $\operatorname{cof}(F)$. We reserve these explicit calculations for later sections, but it is useful to recall at the outset the following two $3 \times 3$ matrix identities:

$$
\operatorname{Det}(A)=\frac{1}{6}\left(\operatorname{Tr}(A)^{3}-3 \operatorname{Tr}\left(A^{2}\right) \operatorname{Tr}(A)+2 \operatorname{Tr}\left(A^{3}\right)\right)
$$

$\operatorname{cof}(A)=\frac{1}{2} I\left(\operatorname{Tr}(A)^{2}-\operatorname{Tr}\left(A^{2}\right)\right)-A^{T} \operatorname{Tr}(A)+A^{T} A^{T}$.

Furthermore, inserting the series expansion for $F$ into the previous matrix identities yields the general fourth order expansions of $\operatorname{Det}(F)$ and $\operatorname{cof}(F)$,

$$
\begin{aligned}
& \operatorname{Det}(F)=1+\epsilon \operatorname{Tr}\left(F_{1}\right)+\epsilon^{2}\left(\operatorname{Tr}\left(F_{2}\right)+\frac{\operatorname{Tr}\left(F_{1}\right)^{2}-\operatorname{Tr}\left(F_{1}^{2}\right)}{2}\right) \\
& +\epsilon^{3}\left(\operatorname{Tr}\left(F_{3}\right)+\frac{\operatorname{Tr}\left(F_{1}\right)^{3}-3 \operatorname{Tr}\left(F_{1}\right) \operatorname{Tr}\left(F_{1}^{2}\right)+2 \operatorname{Tr}\left(F_{1}^{3}\right)}{6}-\operatorname{Tr}\left(F_{1} F_{2}\right)\right. \\
& \left.+\operatorname{Tr}\left(F_{1}\right) \operatorname{Tr}\left(F_{2}\right)\right)+\epsilon^{4}\left(\operatorname{Tr}\left(F_{4}\right)+\operatorname{Tr}\left(F_{1}\right) \operatorname{Tr}\left(F_{3}\right)-\operatorname{Tr}\left(F_{1} F_{3}\right)\right. \\
& +\frac{1}{2}\left(\operatorname{Tr}\left(F_{2}\right)^{2}-\operatorname{Tr}\left(F_{2}^{2}\right)\right)-\operatorname{Tr}\left(F_{1}\right) \operatorname{Tr}\left(F_{1} F_{2}\right)+\operatorname{Tr}\left(F_{1}^{2} F_{2}\right) \\
& \left.+\frac{1}{2}\left(\operatorname{Tr}\left(F_{1}\right)^{2} \operatorname{Tr}\left(F_{2}\right)-\operatorname{Tr}\left(F_{1}^{2}\right) \operatorname{Tr}\left(F_{2}\right)\right)\right)+\mathcal{O}\left(\epsilon^{5}\right)
\end{aligned}
$$

$\operatorname{cof}(F)=I+\epsilon\left(\operatorname{Tr}\left(F_{1}\right) I-F_{1}^{T}\right)+\epsilon^{2}\left(\operatorname{Tr}\left(F_{2}\right) I-F_{2}^{T}\right.$

$$
\begin{aligned}
& \left.+\frac{1}{2}\left(\operatorname{Tr}\left(F_{1}\right)^{2}-\operatorname{Tr}\left(F_{1} F_{1}\right)\right) I-\operatorname{Tr}\left(F_{1}\right) F_{1}^{T}+F_{1}^{T} F_{1}^{T}\right) \\
& +\epsilon^{3}\left(\operatorname{Tr}\left(F_{3}\right) I-F_{3}^{T}+I\left(\operatorname{Tr}\left(F_{2}\right) \operatorname{Tr}\left(F_{1}\right)-\operatorname{Tr}\left(F_{2} F_{1}\right)\right)\right. \\
& \left.-F_{2}^{T} \operatorname{Tr}\left(F_{1}\right)-F_{1}^{T} \operatorname{Tr}\left(F_{2}\right)+F_{2}^{T} F_{1}^{T}+F_{1}^{T} F_{2}^{T}\right) \\
& +\epsilon^{4}\left(\operatorname{Tr}\left(F_{4}\right) I-F_{4}^{T}+\frac{1}{2}\left(\operatorname{Tr}\left(F_{2}\right)^{2}-\operatorname{Tr}\left(F_{2} F_{2}\right)\right)\right. \\
& +\operatorname{Tr}\left(F_{1}\right) \operatorname{Tr}\left(F_{3}\right)-\operatorname{Tr}\left(F_{1} F_{3}\right)-F_{3}^{T} \operatorname{Tr}\left(F_{1}\right)-F_{1}^{T} \operatorname{Tr}\left(F_{3}\right) \\
& \left.-F_{2}^{T} \operatorname{Tr}\left(F_{2}\right)+F_{2}^{T} F_{2}^{T}+F_{3}^{T} F_{1}^{T}+F_{1}^{T} F_{3}^{T}\right)+\mathcal{O}\left(\epsilon^{5}\right) .
\end{aligned}
$$

\section{A. The flat state}

Substituting the $\sigma$ expansion into the equations of equilibrium and expanding to $\mathcal{O}\left(\epsilon^{0}\right)$, we see that the flat state we are perturbing around must satisfy

$$
\nabla \cdot \sigma_{0}=\mathbf{0}, \quad \operatorname{Det}(I)=1
$$

in the bulk, and

$$
\left.\sigma_{0} \cdot \hat{\mathbf{z}}\right|_{z=a}=\mathbf{0} \quad \text { and }\left.\quad \sigma_{0} \cdot \hat{\mathbf{z}}\right|_{z=0^{+}}=\left.\sigma_{0} \cdot \hat{\mathbf{z}}\right|_{z=0^{-}} \cdot
$$

on the boundaries. Inserting the $F$ expansion into the definition of $\sigma$, we see that

$$
\sigma_{0}=\tilde{\mu} \operatorname{diag}\left(1 / \tilde{g}^{2}-P_{0}, 1 / \tilde{g}^{2}-P_{0}, \tilde{g}^{4}-P_{0}\right),
$$

which indeed is divergence free provided $P_{0}$ is constant, meaning both bulk equations are satisfied. The boundary conditions at $z=a$ and $z=0$ then determine the flatstate pressure as

$$
P_{0}=\tilde{g}^{4}
$$

\section{B. First order perturbation theory}

The $\mathcal{O}(\epsilon)$ terms in the bulk equations are:

$$
\nabla \cdot \sigma_{1}=\mathbf{0}, \quad \operatorname{Tr}\left(F_{1}\right)=\nabla \cdot \mathbf{u}_{1}=0,
$$


the first order boundary conditions are,

$$
\begin{aligned}
\left.\sigma_{1} \cdot \hat{\mathbf{z}}\right|_{z=a} & =-\left.2 L_{0} u_{1 z} \hat{\mathbf{z}}\right|_{z=a} \\
\left.\sigma_{1} \cdot \hat{\mathbf{z}}\right|_{z=0^{+}} & =\left.\sigma_{1} \cdot \hat{\mathbf{z}}\right|_{z=0^{-}} \\
\left.\mathbf{u}_{1}\right|_{z=0^{+}} & =\left.\mathbf{u}_{1}\right|_{z=0^{-}},
\end{aligned}
$$

and the first order amplitude equation is

$$
\left.\left\langle u_{1 z}^{2}\right\rangle\right|_{z=a}=1 \text {. }
$$

Inserting the expansion for $\operatorname{cof}(F)$ into the general expression for $\sigma$, we see that

$$
\sigma_{1}=\tilde{\mu}\left(F_{1} G^{-1} G^{-T}+P_{0} F_{1}^{T}-P_{1} I\right) .
$$

The first order energy coefficient is $E_{1}=\int W_{1} \mathrm{~d} V$, which we expect to be zero, as it is the first order deviation away from the equilibrium flat state. More explicitly, inserting inserting the expansion for $\operatorname{Det}(F)$ into $W$ gives $W_{1}=$ $\sigma_{0} F_{1}=\sigma_{0} \nabla \mathbf{u}_{1}$, which is not zero, but which gives zero for $E_{1}$ after integration by parts. The energy coefficient we are ultimately seeking to calculate is $E_{2}=\int W_{2} \mathrm{~d} V$, so we expand $W$ to second order, giving

$$
W_{2}=\frac{1}{2} \tilde{\mu}\left(\operatorname{Tr}\left(F_{1} \cdot G^{-1} \cdot G^{-T} \cdot F_{1}^{T}\right)-P_{0} \operatorname{Tr}\left(F_{1} \cdot F_{1}\right)\right),
$$

where we have used incompressibility to eliminate the $P_{1} \operatorname{Tr}\left(F_{1}\right)$ term, and omitted a $\sigma_{0} F_{2}$ term as it will also integrate to zero in $E_{2}$.

For the stripe pattern, the first order displacement field has a single Fourier component $u_{1 z} \sim f_{z}(z) \cos (k x)$, which requires sympathetically varying fields of the form

$$
\begin{aligned}
& \mathbf{u}_{1}=\left(\begin{array}{c}
f_{x}(z) \sin (k x) \\
0 \\
f_{z}(z) \cos (k x)
\end{array}\right) \\
& P_{1}=f_{p}(z) \cos (k x),
\end{aligned}
$$

so first order correction to the deformation gradient is

$$
F_{1}=\nabla \mathbf{u}_{1}=\left(\begin{array}{ccc}
k f_{x} \cos (k x) & 0 & f_{x}^{\prime} \sin (k x) \\
0 & 0 & 0 \\
-k f_{z} \sin (k x) & 0 & f_{z}^{\prime} \cos (k x)
\end{array}\right) \text {. }
$$

Substituting these forms for $F_{1}$ and $\sigma_{1}$ into the bulk equations, the first order incompressibility equation is:

$$
k f_{x}+f_{z}^{\prime}=0
$$

and the first order mechanical equilibrium equations are:

$$
\begin{array}{r}
\tilde{g}^{2} k f_{p}-k^{2} f_{x}+\tilde{g}^{6} f_{x}^{\prime \prime}=0 \\
k^{2} f_{z}+\tilde{g}^{2} f_{p}^{\prime}-\tilde{g}^{6} f_{z}^{\prime \prime}=0 .
\end{array}
$$

These can be algebraically solved for $f_{p}$ and $f_{x}$,

$$
\begin{aligned}
& f_{p}(z)=\frac{\tilde{g}^{6} f_{z}^{\prime \prime \prime}-k^{2} f_{z}^{\prime}}{\tilde{g}^{2} k^{2}} \\
& f_{x}(z)=-\frac{f_{z}^{\prime}}{k}
\end{aligned}
$$

then substituting these results into the second mechanical equilibrium equation yields a fourth order differential equation for $f_{z}$ :

$$
k^{4} f_{z}-\left(1+\tilde{g}^{6}\right) k^{2} f_{z}^{\prime \prime}+\tilde{g}^{6} f_{z}^{(4)}=0,
$$

which is solved by the form,

$f_{z}= \begin{cases}e^{-k z / g^{3}} A_{1}+e^{k z / g^{3}} A_{2}+e^{-k z} A_{3}+e^{k z} A_{4} & z>0, \\ e^{k z}\left(B_{1}+z B_{2}\right) & z<0\end{cases}$

where $A_{i}$ and $B_{i}$ are constants of integration, and we have excluded solutions that diverge as $z \rightarrow-\infty$. The values of $A_{i}, B_{i}$ and $L_{0}$ must be found from the boundary conditions (34-37). The normal stress condition on the top surface (the $z$ component of (34)) is a linear equation for $L_{0}$, solved in terms of the $A_{i}$ and $B_{i}$ as:

$$
L_{0}=-\left.\frac{\sigma_{1 z z}}{2 u_{1 z}}\right|_{z=a} .
$$

The remaining six boundary conditions (amplitude, surface shear stress, $2 \times$ interfacial stress continuity, $2 \times$ interfacial displacement continuity) are six linear equations in the form $M\left(A_{1}, A_{2}, A_{3}, A_{4}, B_{1}, B_{2}\right)=$ $(\sqrt{2}, 0,0,0,0,0)$, with $M$ of the form

$$
\left(\begin{array}{cccccc}
e^{-k a / g^{3}} & e^{k a / g^{3}} & e^{-k a} & e^{k a} & 0 & 0 \\
e^{-k a / g^{3}} b & e^{k a / g^{3}} b & 2 e^{-k a} g^{6} & 2 e^{k a} g^{6} & 0 & 0 \\
-2 g^{3} \eta & 2 g^{3} \eta & -b \eta & b \eta & -2 g^{2} & 0 \\
b \eta & b \eta & 2 g^{6} \eta & 2 g^{6} \eta & -2 g^{2} & -2 g^{2} / k \\
1 & 1 & 1 & 1 & -1 & 0 \\
1 & -1 & g^{3} & -g^{3} & g^{3} & g^{3} / k
\end{array}\right)
$$

where $b=1+g^{6}$. The non-zero source on the right of this matrix equation arises from the amplitude equation, and ensures that there is always a solution, of the form $\left(A_{1}, A_{2}, A_{3}, A_{4}, B_{1}, B_{2}\right)=M^{-1}(\sqrt{2}, 0,0,0,0,0)$. This solution corresponds, for each value of $g$, to a solution with the small but prescribed amplitude $\epsilon$ imposed by the external surface stress $L_{0}$. The resulting expressions for $A_{i}$ and $L_{0}$ are rather cumbersome, so we do not display them here, though they are included in our computer-algebra verification discussed in the next sections. The threshold for spontaneous instability arises at the critical degree of growth $g^{*}$ for which this solution has $L_{0}=0$, indicating a solution that arises without external stress, and coinciding with a sign change in $L$ indicating its changing role from enhancing to reducing instability amplitude. To find the threshold value $g^{*}$, one must simply solve the additional threshold equation $L_{0}=0$ for $g$. This equation cannot be solved analytically, but is straightforward to solve numerically at any numerical values of $k$ and $\eta$. Fig. 4 characterizes the results of this stability analysis. Fig. 4A shows the threshold $g^{*}$ as a function of wavelength, $\lambda=2 \pi / k$, for different $\eta$ and Figs $4 \mathrm{~B}$ and $\mathrm{C}$ show the threshold and the wavelength of the first unstable mode, obtained by numerically minimizing Fig. 4A over wavelengths. As expected, 

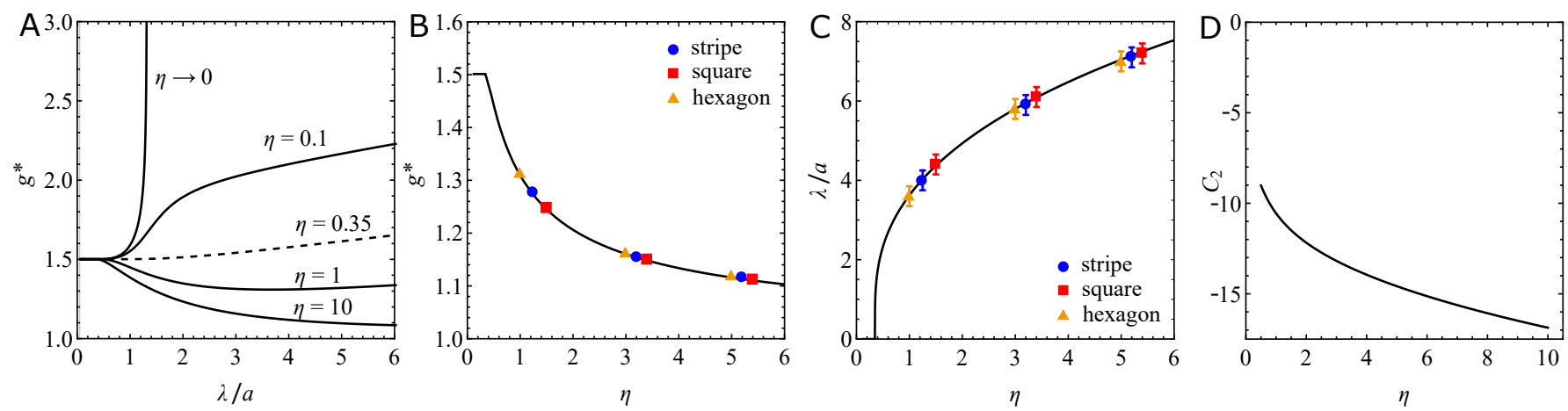

FIG. 4. (A) Threshold value of growth $g$ as a function of wavelength at different values of stiffness ratio, $\eta$. (B) and (C) threshold growth and the wavelength of the first unstable mode. Data points show the numerical results. (D) The second order energy coefficient $C_{2}$, of the first unstable mode as a function of the stiffness ratio, $\eta$.

for stiff layers the critical wavelength grows with layer stiffness, and the buckling mode is a form of Euler buckling constrained by the substrate. In the other extreme, for $\eta \lesssim 0.35$ the first unstable mode has zero wavelength at $g=1.501 \ldots$, the hallmark of the pathological Biot surface instability. From previous work, we know that this Biot instability is never observed, because it is always preceded by the non-linear sulcus/crease forming instability which, in neo-Hookeans occurs at $g^{*} \approx 1.34$. In our system, this nonlinear instability occurs before the linear instability for $\eta \lesssim 0.86$, which is thus the limit of validity of our perturbative approach.

Although the above treatment deals with stripe patterns, first order square patterns and hexagonal patterns are simple linear combinations of this underlying stripe patterns, and hence described by the same elastic solution. For the square pattern we have

$$
\begin{aligned}
& \mathbf{u}=\frac{\epsilon}{\sqrt{2}}\left(\begin{array}{c}
f_{x}(z) \sin (k x) \\
f_{x}(z) \sin (k y) \\
f_{z}(z)(\cos (k x)+\cos (k y))
\end{array}\right) \\
& P=P_{0}(z)+\frac{\epsilon}{\sqrt{2}} f_{p}(z)(\cos (k x)+\cos (k y))
\end{aligned}
$$

and for the hexagonal pattern we have

$$
\begin{aligned}
& \mathbf{u}=\frac{\epsilon}{\sqrt{3}}\left(\begin{array}{c}
f_{x}(z)\left(\sin (k x)+\frac{1}{2}\left(\sin \left(k x_{1}\right)-\sin \left(k x_{2}\right)\right)\right) \\
f_{x}(z) \frac{\sqrt{3}}{2}\left(\sin \left(k x_{1}\right)+\sin \left(k x_{2}\right)\right) \\
f_{z}(z)\left(\cos (k x)+\cos \left(k x_{1}\right)+\cos \left(k x_{2}\right)\right)
\end{array}\right) \\
& P=P_{0}(z)+\frac{\epsilon}{\sqrt{3}} f_{p}(z)\left(\cos (k x)+\cos \left(k x_{1}\right)+\cos \left(k x_{2}\right)\right),
\end{aligned}
$$

where the overall prefactors have been chosen so that all three patterns have the same root-mean-squared amplitude. The value of $E_{2}$ is the same for each pattern, as the integrand underlying $E_{2}$ is quadratic in the elastic fields, so cross-terms between different Fourier components in the square/hexagonal patterns integrate to zero. As expected, $E_{2}$ vanishes at $g^{*}$, indicating a transition from stability to instability. We calculate $C_{2}$ as

$$
C_{2}=\left.\frac{\partial E_{2}}{\partial g}\right|_{g=g *},
$$

so that, to quadratic order, the energy can be written as $E=E_{0}+C_{2}\left(g-g^{*}\right) \epsilon^{2}$. Using computer algebra, we are able to calculate this derivative analytically, then substitute in numerical threshold values for $g^{*}$ and $k$, leading to a numerical value of $C_{2}$ for each value of $\eta$, which is plotted in Fig. 4D.

To verify our results, a finite element numerical simulation is performed to find the critical growth and wavelength (Dots in Figs 4B and C), showing a good agreement with the theory regardless of the pattern as we have claimed. The simulation was done with a symmetryconstrained domain which only allows a chosen wavelength and pattern (See Subsections V E-V G for details).

\section{Second order perturbation theory}

The $\mathcal{O}\left(\epsilon^{2}\right)$ terms in the bulk equations are:

$$
\nabla \cdot \sigma_{2}=\mathbf{0}, \quad \operatorname{Tr}\left(F_{2}\right)=\frac{1}{2} \operatorname{Tr}\left(F_{1}^{2}\right),
$$

the second order boundary conditions are,

$$
\begin{aligned}
\left.\sigma_{2} \cdot \hat{\mathbf{z}}\right|_{z=a} & =-\left.2\left(L_{1} u_{1 z}+L_{0} u_{2 z}\right) \hat{\mathbf{z}}\right|_{z=a} \\
\left.\sigma_{2} \cdot \hat{\mathbf{z}}\right|_{z=0^{+}} & =\left.\sigma_{2} \cdot \hat{\mathbf{z}}\right|_{z=0^{-}} \\
\left.\mathbf{u}_{2}\right|_{z=0^{-}} & =\left.\mathbf{u}_{2}\right|_{z=0^{+}},
\end{aligned}
$$

and the second order amplitude equation is

$$
\left.\left\langle u_{1 z} u_{2 z}\right\rangle\right|_{z=a}=0 .
$$

Again expanding $\sigma$ using the general expansion of $\operatorname{cof}(F)$, the expression of $\sigma_{2}$ is

$\sigma_{2}=\tilde{\mu}\left(F_{2} G^{-1} G^{-T}-P_{2} I+P_{1} F_{1}^{T}+P_{0}\left(F_{2}^{T}-F_{1}^{T} F_{1}^{T}\right)\right)$.

These equations must be solved for $\mathbf{u}_{2}, P_{2}$ and $L_{1}$. The equations are linear in these variables but, as seen in 
(53) and (58), they also contain quadratic source terms in the first order fields. These quadratic terms introduce Fourier components corresponding to all the sums and differences of the $k$ vectors in the first order solutions, so at second and higher order the different patterns produce solutions that are not simple linear combinations of each other, and indeed each equation becomes the sum of several different in-plane Fourier modes. Our approach is to substitute trial solutions for each pattern that are sums of all permissible in-plane Fourier modes but with unknown $z$ dependence, then require each mode in each equation to vanish separately. This produces a long set of bulk and boundary equations, which are algebraically tedious but mechanically simple to solve for the unknown $z$ variation in the second order fields.

After finding the fields, we can integrate the third order energy density coefficient,

$$
\begin{aligned}
W_{3}=\tilde{\mu} & \left(\operatorname{Tr}\left(F_{1} \cdot G^{-1} \cdot G^{-T} \cdot F_{2}^{T}\right)\right. \\
& \left.-P_{0}\left(\frac{1}{3} \operatorname{Tr}\left(F_{1}^{3}\right)-\operatorname{Tr}\left(F_{1} F_{2}\right)\right)\right)
\end{aligned}
$$

to find the coefficient $C_{3}$ that we are seeking. As earlier, we have omitted a $\sigma_{0} F_{3}$ term in this expansion, as it will integrate to zero in $C_{3}$. As with $C_{2}$, the integration can be done using computer algebra, but our final result is the evaluation of this algebraic expression at the numerically found threshold for instability.

\section{Third order perturbation theory}

The $\mathcal{O}\left(\epsilon^{3}\right)$ terms in the bulk equations are:

$$
\nabla \cdot \sigma_{3}=\mathbf{0}, \quad \operatorname{Tr}\left(F_{3}\right)=\operatorname{Tr}\left(F_{1} F_{2}\right)-\frac{1}{3} \operatorname{Tr}\left(F_{1}^{3}\right),
$$

the third order boundary conditions are,

$$
\begin{aligned}
\left.\sigma_{3} \cdot \hat{\mathbf{z}}\right|_{z=a} & =-\left.2\left(L_{2} u_{1 z}+L_{1} u_{2 z}+L_{0} u_{3 z}\right) \hat{\mathbf{z}}\right|_{z=a} \\
\left.\sigma_{3} \cdot \hat{\mathbf{z}}\right|_{z=0^{+}} & =\left.\sigma_{3} \cdot \hat{\mathbf{z}}\right|_{z=0^{-}} \\
\left.\mathbf{u}_{3}\right|_{z=0^{-}} & =\left.\mathbf{u}_{3}\right|_{z=0^{+}}
\end{aligned}
$$

and the third order amplitude equation is

$$
\left.\left\langle u_{1 z} u_{3 z}+\frac{1}{2} u_{2 z}^{2}\right\rangle\right|_{z=a}=0 .
$$

Again expanding $\sigma$ using the general expansion of $\operatorname{cof}(F)$, the expression of $\sigma_{3}$ is

$$
\begin{aligned}
& \sigma_{3}=\tilde{\mu}\left(F_{3} G^{-1} G^{-T}-P_{3} I+P_{2} F_{1}^{T}+P_{1}\left(F_{2}^{T}-F_{1}^{T} F_{1}^{T}\right)\right. \\
& \left.+P_{0}\left(\frac{1}{3} \operatorname{Tr}\left(F_{1}^{3}\right) I+F_{3}^{T}+F_{1}^{T} \operatorname{Tr}\left(F_{2}\right)-F_{2}^{T} F_{1}^{T}-F_{1}^{T} F_{2}^{T}\right)\right) .
\end{aligned}
$$

These equations must be solved for $\mathbf{u}_{3}, P_{3}$ and $L_{2}$. Again the equations are linear in the solution variables, but this time the "source" terms are cubic terms of the first and second order fields, so there will be in-plane Fourier components corresponding to all three-fold sums of initial $k$ vectors, leading to a very large total number of equations to solve. After finding the fields, we can integrate the fourth order energy density coefficient,

$$
\begin{aligned}
& W_{4}=\tilde{\mu}\left[\operatorname{Tr}\left(\frac{1}{2} F_{2} G^{-1} G^{-T} F_{2}^{T}+F_{1} G^{-1} G^{-T} F_{3}^{T}\right)\right. \\
& \left.-P_{0}\left(\operatorname{Tr}\left(F_{1}^{2} F_{2}\right)-\operatorname{Tr}\left(F_{1} F_{3}\right)-\frac{1}{2}\left(\operatorname{Tr}\left(F_{2}\right)^{2}+\operatorname{Tr}\left(F_{2}^{2}\right)\right)\right)\right],
\end{aligned}
$$

to find the coefficient $C_{4}$ that we are seeking. As earlier, we have omitted a $\sigma_{0} F_{4}$ term in this expansion, as it will integrate to zero in $C_{4}$. As with $C_{2}$ and $C_{3}$, the integration can be done using computer algebra, but our final result is the evaluation of the resulting algebraic expression at the numerically found threshold for instability.

\section{E. Stripe pattern results}

As noted in the previous two sections, the second and third order fields are expected to require all Fourier components that are two-fold and three-fold sums of the $k$ vectors of the first order field. In the case of a stripe pattern, this leads us to consider the form:

$$
\begin{aligned}
& u_{x}(x, z)=\epsilon f_{x, 1,1}(z) \sin (k x)+\epsilon^{2}\left(f_{x, 2,1}(z) \sin (2 k x)\right) \\
& +\epsilon^{3}\left(f_{x, 3,1}(z) \sin (k x)+f_{x, 3,2}(z) \sin (3 k x)\right)+\mathcal{O}\left(\epsilon^{4}\right) \\
& u_{z}(x, z)=\epsilon f_{z, 1,1}(z) \cos (k x) \\
& +\epsilon^{2}\left(f_{z, 2,1}(z)+f_{z, 2,2}(z) \cos (2 k x)\right) \\
& +\epsilon^{3}\left(f_{z, 3,1}(z) \cos (k x)+f_{z, 3,2}(z) \cos (3 k x)\right)+\mathcal{O}\left(\epsilon^{4}\right) \\
& P(x, z)=P_{0}(z)+\epsilon f_{p, 1,1}(z) \cos (k x) \\
& +\epsilon^{2}\left(f_{p, 2,1}(z)+f_{p, 2,2}(z) \cos (2 k x)\right) \\
& +\epsilon^{3}\left(f_{p, 3,1}(z) \cos (k x)+f_{p, 3,2}(z) \cos (3 k x)\right)+\mathcal{O}\left(\epsilon^{4}\right)
\end{aligned}
$$

where the $f_{x, m, n}(z), f_{z, m, n}(z)$ and $f_{p, m, n}(z)$ indicate unknown functions describing the $z$ variation of the $n^{\text {th }}$ Fourier component (in $u_{x}, u_{y}$ and $P$ respectively) at $m^{\text {th }}$ order in $\epsilon$, so in the notation from the first order perturbation theory $f_{x, 1,1}=f_{x}, f_{z, 1,1}=f_{z}$ and $f_{p, 1,1}=f_{p}$. Although we have found these first order fields by hand, we now use the computer algebra package Mathematica to solve at higher order.

More precisely, we first substitute these forms into the bulk equations and boundary/amplitude conditions, expand the equations in $\epsilon$, separate the first, second and third order equations (as we did by hand in the previous two sections) and then further separate these equations into their different Fourier components, yielding a long list of bulk and boundary equations at each order in $\epsilon$. Reminiscent of first order, at each successive order $\epsilon^{m}$ the equations arising from the bulk equations are a linear algebraic/differential set of equations for the $f_{x, m, n}(z), f_{z, m, n}(z)$ and $f_{p, m, n}(z)$, which Mathematica is 
able to solve analytically as a set using "DSolve" command, thereby introducing a number of constants of integration.

We next substitute these solutions into the elastic energy, expand in $\epsilon$ and explicitly integrate over the total volume, to find an exact expression for each energy coefficient $E_{0} \ldots E_{4}$, though in terms of the as-yet unknown constants of integration. Fortunately the equations arising from the boundary/amplitude condition form a linear set of equations for the constants of integration, with a single unique solution which Mathematica is again able to find analytically using "Solve" command. Finally, we substitute these expressions for the constants into $E_{0} \ldots E_{4}$, and evaluate the resulting expressions at the numerically characterized point of instability to find $C_{3}$ and $C_{4}$. We provide a Mathematica workbook as supplementary information ${ }^{\dagger}$, which includes the full algebraic forms of the solution, verifies the it solves the equations, and computes the resulting energy coefficients.

As anticipated, the third order coefficient $C_{3}$ vanishes identically for the stripe pattern upon conducting the inplane integration. We show the energy coefficients $C_{4}$ for the stripe pattern as a function of $\eta$, (evaluated at each $\eta$ at the value of $g^{*}$ and $k$ of the first unstable mode) in Fig. $5 \mathrm{~A}$. The $C_{4}$ coefficient is positive except when $\eta<0.78$, where the energy series expansion (16) will need to be evaluated to higher orders to find the amplitude. This means that that the stripe pattern will be supercritical for stiff layer but subcritical in very soft layers. However, since this transition occurs below the sulcification limit at $\eta_{s}=0.86$, it cannot be observed in practice. Using the results in (18-19), we can predict the amplitude and the energy for the stripe pattern close to threshold, and these are plotted for $\eta=3$ in Figs $5 \mathrm{C}$ and D.

We performed a numerical finite element analysis to verify these theoretical predictions. To do so, we used large-strain fully non-linear finite elements, but constrained the pattern to stripes by choosing the domain of the simulation as a rectangular unit cell, with the longer side equal to predicted wavelength and a periodic boundary condition. The simulated pattern is shown in Fig. $5 \mathrm{~B}$, and the simulated amplitude and energy are compared with the theoretical prediction in Fig. 5C, showing a good agreement (with no fitting parameters of any kind) especially close to the threshold, and thereby lending confidence to our computer algebra. No hysteresis loop is observed numerically as expected from a supercritical instability.

\section{F. Square pattern results}

For the square pattern, the forms of the displacement and pressure fields become long, so we display them in Appendix B. However, we are again able to substitute these forms into the energy/equations, and find a complete third order solution in the same manner as for stripes. Again, the coefficient $C_{3}$ vanishes upon in-plane
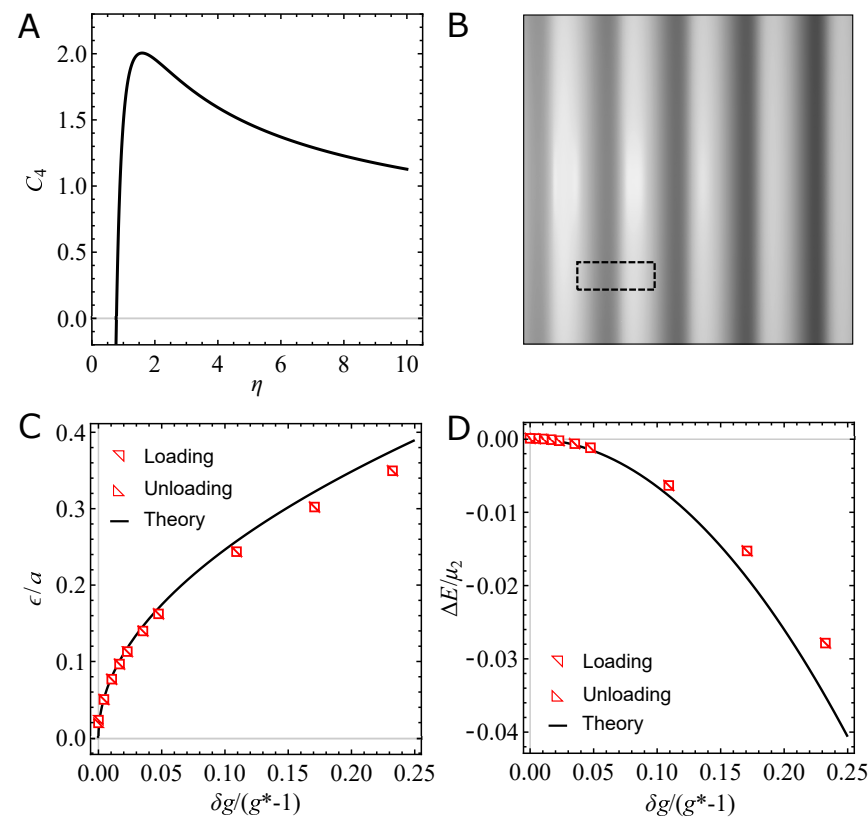

FIG. 5. (A) The energy coefficient $C_{4}$. (B) The numerically simulated pattern at $g=1.18$ for $\eta=3$. Dashed lines show a simulation unit cell. (C) Amplitude and (D) energy per slab volume as a function of growth from the threshold, $\delta g=g-g^{*}$, for stripe patterns at $\eta=3$.

integration of the energy, and we plot the form of the coefficient $C_{4}$ as a function of $\eta$ in Fig. 6. In this case $C_{4}$ is also positive for stiff layers, meaning the instability is supercritical, and we can again predict the amplitude and energy of instability close to the threshold. However, in this case, $C_{4}$ becomes negative for $\eta<2.23$ indicating a transition to a subcritical instability at this value of $\eta$, which is above the sulcification point, and therefore in the domain of validity of the theory. Below this point, we cannot estimate the amplitude or energy of the instability (as this would require $C_{6}$ ) but we can be confident the instability is subcritical.

To test these predictions, we again perform numerical finite element calculations, this time selecting $\eta=1.5,3$ and 10, to span the predicted supercritical to subcritical transition. In this case, we constrained the finite elements to a square pattern by using a square unit cell with the side equal to the predicted wavelength. As seen in Figs $6 \mathrm{~B}-\mathrm{E}$, for $\eta=10$ the transition is indeed supercritical, and in good agreement with the theoretical prediction. For $\eta=1.5$, the transition is indeed subcritical, as predicted. For $\eta=3$, the transition is supercritical as predicted, but the actual amplitude and energy are in poor agreement, which we attribute to the fact that $C_{4}$ is almost zero (as it is close to the positive-negative transition) so higher orders are required for an accurate prediction even close to threshold. 

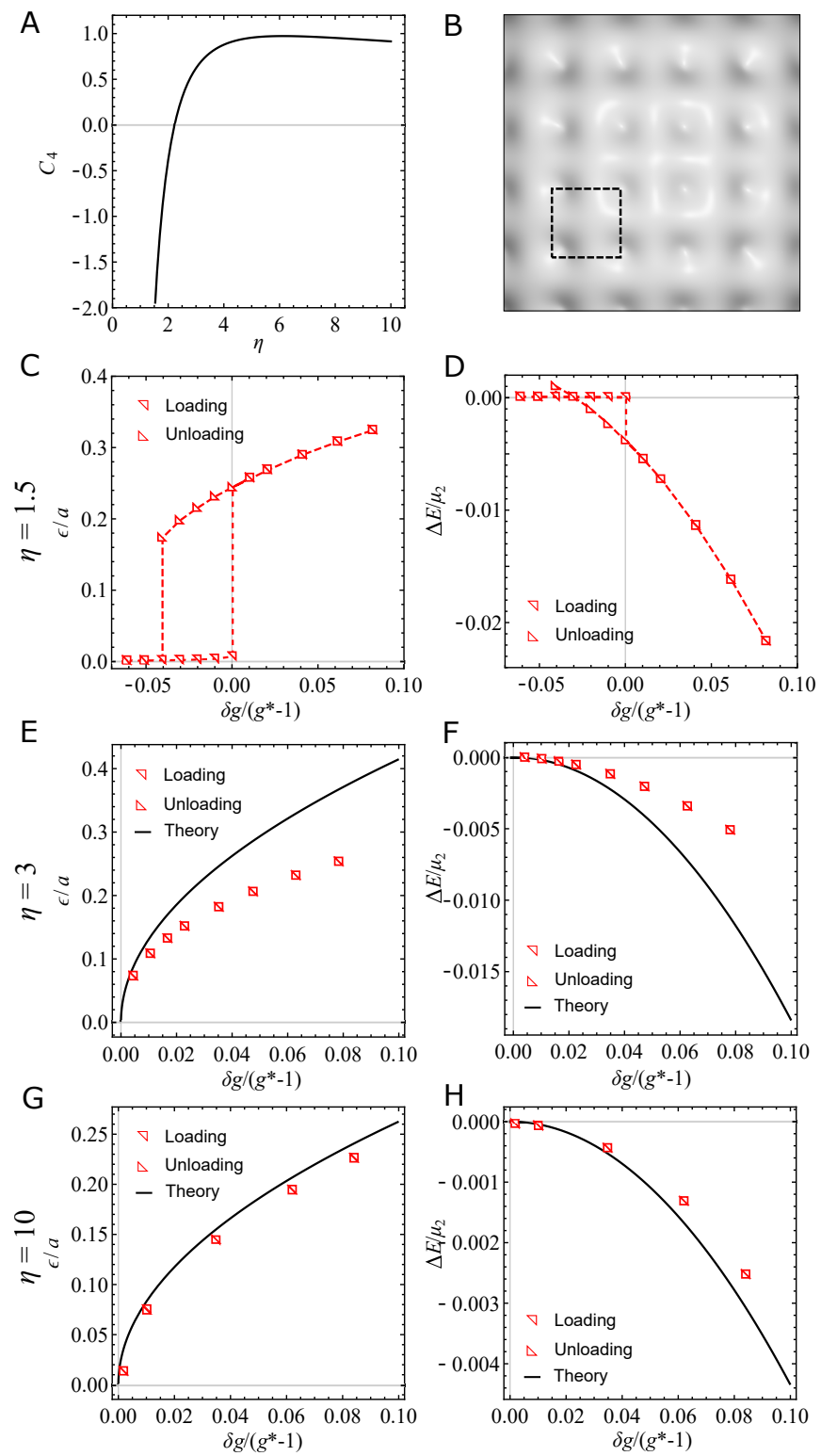

FIG. 6. (A) The energy coefficient $C_{4}$. (B) The numerically simulated pattern at $g=1.18$ for $\eta=3$. Dashed lines show a simulation unit cell. (C-H) Amplitude and energy per slab volume as a function of growth from the threshold, $\delta g=g-g^{*}$, for square patterns at $\eta=1.5,3$ and 10 .

\section{G. Hexagonal pattern results}

As for the square pattern, the forms of the displacement and pressure fields for the hexagonal pattern become rather long, so we display them in Appendix B. However, we are again able to substitute these forms into the energy/equations, and find a complete third-order solution in the same manner as for stripes and squares. We summarize the energy coefficient $C_{3}$ and $C_{4}$ in Fig. 7A,B. In this case, the coefficient $C_{3}$ does not vanish, but is positive for all $\eta$, leading us to conclude that the hexagonal instability is subcritical and will produce patterns of
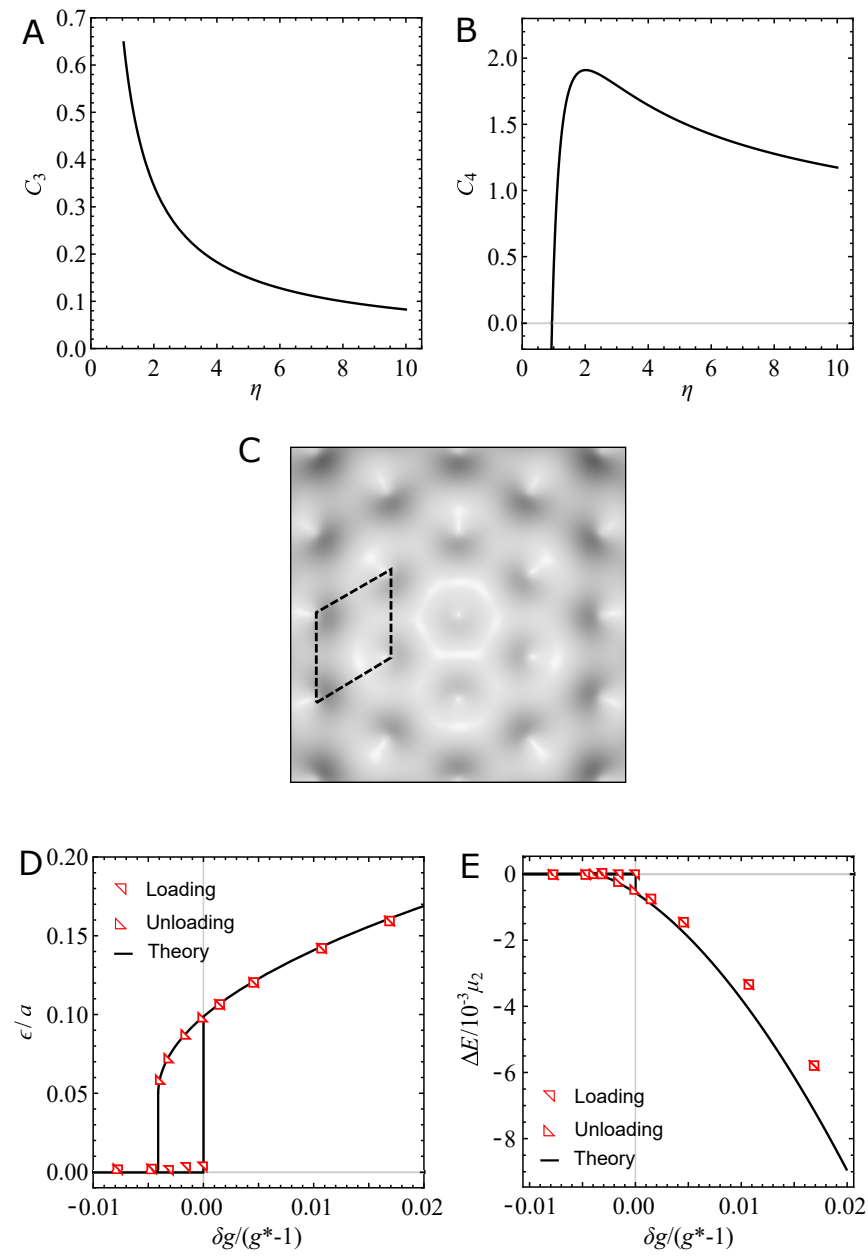

FIG. 7. (A and B) The energy coefficient $C_{4}$. (C) The numerically simulated pattern at $g=1.18$ for $\eta=3$. Dashed lines show a simulation unit cell. (D) Amplitude and (E) Energy per slab volume as a function of growth from the threshold, $\delta g=g-g^{*}$, for hexagonal patterns at $\eta=3$.

hexagonal dents, as previously observed in experiment. The coefficient $C_{4}$ is similar in magnitude to that for the stripe patterns, and changes sign from positive to negative at $\eta=0.95$, but since the instability is already subcritical this is of little consequence.

In the region where $C_{3}>0$ and $C_{4}>0$, we are able to estimate the energy and amplitude after instability, though we emphasize that in this case it is only an estimate, since the subcritical jump produces a non-small amplitude that is beyond the validity of perturbation theory. We again plot these predictions at $\eta=3$ in Figs 7D and $\mathrm{E}$, and compare them to fully non-linear finite element solutions. These finite element calculations confirm that the hexagonal instability is subcritical, and agree with the predicted subcritical amplitude and energy remarkably well. 

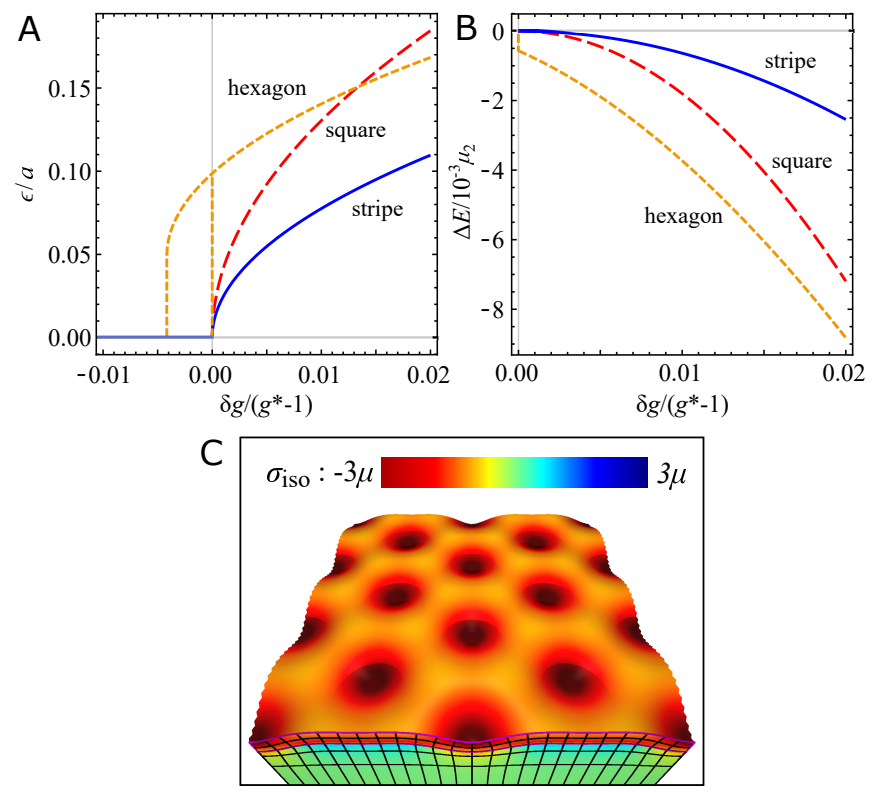

FIG. 8. (A) Amplitude and (B) energy per unit volume as a function of growth from the threshold, $g-g^{*}$, for each pattern at $\eta=3$. (C) The hexagonal pattern in a large square domain for $\eta=3$ at $g=1.24$.

\section{H. Summary of instability in a neo-Hookean bilayer}

To summarize our results, we compare the predicted amplitude and energy close to the threshold at $\eta=3$ in Fig 8. As anticipated, the hexagonal pattern is the only subcritical pattern, and thus has the lowest energy close to threshold, in accordance with our earlier symmetry argument. We further verify this conclusion by conducting a final finite element calculation on a large square domain with periodic boundary conditions, which nevertheless spontaneously produced a hexagonal pattern close to threshold (Fig 8C).

\section{COMPRESSIBLE NEO-HOOKEAN LAYER/SUB-STRATE: MANIPULATING $C_{3}$}

For hexagonal patterns, the sign of $C_{3}$ determines whether the pattern forms as bumps or dents, so a key motivation for actually calculating the energy coefficients is to determine this sign. For an incompressible layer on an incompressible substrate, we found in the previous section that $C_{3}$ remains positive for all stiffness ratios, leading to patterns of dents. We now look at varying another parameter, the bulk modulus of the system, and show that this allows a sign change for $C_{3}$ and hence a change from dents to bumps. More precisely, we consider a system in which both layer and substrate are compressible, with each having same ratio between their bulk and shear modulus, given by $K \equiv \kappa / \mu$. We then perform a series-based energy calculation similar to the previous section but with a compressible version of the neo-Hookean energy density,

$$
W=\frac{1}{2} \tilde{\mu}\left[\frac{\operatorname{Tr} B_{G}}{J^{2 / 3}}-3+K(J-1)^{2}\right],
$$

where $J=\operatorname{Det}(F)$ and $B_{G}=F \cdot G^{-1} \cdot G^{-T} \cdot F^{T}$. Minimizing this energy with respect to $\mathbf{u}$ gives the same equation of mechanical equilibrium, $\nabla \cdot \sigma=\mathbf{0}$, and the same boundary conditions (3) and (4) as before, but with a new compressible PK1 stress tensor:

$$
\sigma=\tilde{\mu}\left[\frac{1}{J^{2 / 3}}\left(B_{G}-\frac{\operatorname{Tr} B_{G}}{3} I\right)+K J(J-1) I\right] F^{-T} .
$$

However, as we will see in the next subsection, in the compressible case there is a a uniform displacement of the top surface even in the flat state solution, so we must modify the form of the amplitude constraint to:

$$
\sqrt{\left.\left\langle\left(u_{z}-\left\langle u_{z}\right\rangle\right)^{2}\right\rangle\right|_{z=a}}=|\epsilon| \text {. }
$$

The total energy, with the Lagrange multiplier term, is thus

$$
E=E_{e l}+\left.L\left\langle\left(u_{z}-\left\langle u_{z}\right\rangle\right)^{2}-\epsilon^{2}\right\rangle\right|_{z=a},
$$

and the modified top-surface stress boundary condition is

$$
\left.\left(\sigma \cdot \hat{\mathbf{z}}+2 L\left(u_{z}-\left\langle u_{z}\right\rangle\right) \hat{\mathbf{z}}\right)\right|_{z=a}=\mathbf{0} .
$$

\section{A. The flat state}

As in the incompressible case, we first solve for the flat base state, which must satisfy

$$
\nabla \cdot \sigma_{0}=\mathbf{0},
$$

subjected to the same boundary conditions as the incompressible system. This time $F_{0} \neq I$ as the mechanical equilibrium is satisfied by a uniform deformation in the thin layer in response to the growth,

$$
\begin{aligned}
\mathbf{u}_{0}(x, y, z) & =(0,0,(\tilde{\gamma}-1) z) \\
F_{0} & =\operatorname{diag}(1,1, \tilde{\gamma}),
\end{aligned}
$$

where the constant $\tilde{\gamma}$ is unity in the substrate:

$$
\tilde{\gamma}= \begin{cases}\gamma & 0<z<a \\ 1 & z<0\end{cases}
$$

The boundary condition at the free surface $\left.\sigma_{0} \cdot \hat{\mathbf{z}}\right|_{z=a}=0$ gives

$$
(\gamma-1) K+\frac{2\left(g^{6} \gamma^{2}-1\right)}{3 g^{2} \gamma^{5 / 3}}=0
$$

which dictates the relationship between $\gamma, g$ and $K$. Eqn. (77) cannot be solved analytically for $\gamma$, but we can easily evaluate $\gamma$ numerically for given $g$ and $K$, as shown in Fig. 9, to determine the optimal degree of compression in any given system. 


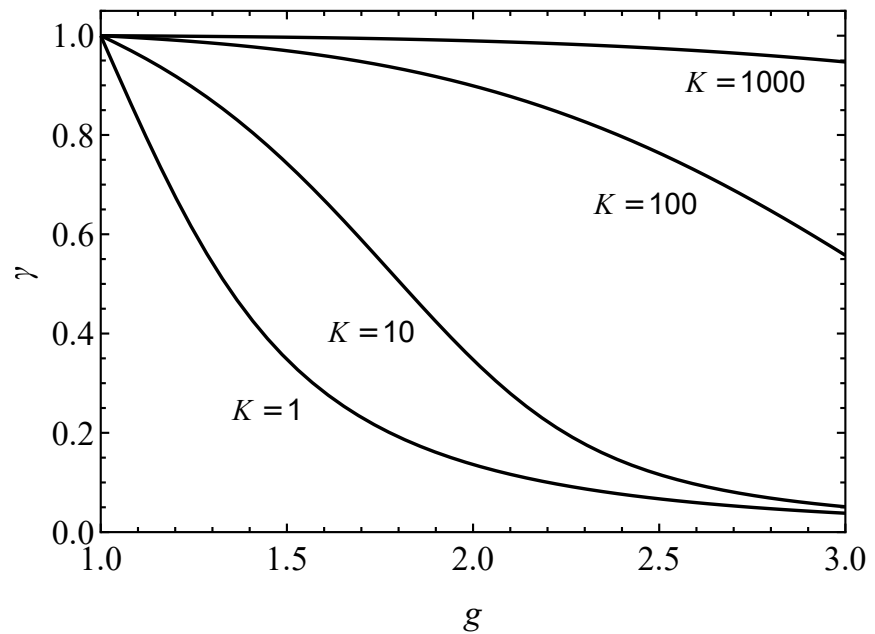

FIG. 9. The flat state deformation $\gamma$ as a function of growth, $g$, for different bulk modulus to shear modulus ratio, $K$.

\section{B. First order perturbation theory}

The bulk equation at $\mathcal{O}(\epsilon)$, again, is

$$
\nabla \cdot \sigma_{1}=\mathbf{0}
$$

For the stripe pattern (with the same form of $\mathbf{u}_{1}$ as the incompressible system, eqn. (39)) the mechanical equilibrium condition give coupled differential equations for $f_{x}$ and $f_{z}$ :

$$
\begin{array}{r}
k^{2} g_{1} f_{x}+k g_{2} f_{z}^{\prime}-9 \tilde{\gamma}^{2} \tilde{g}^{6} f_{x}^{\prime \prime}=0 \\
9 \tilde{\gamma}^{2} k^{2} f_{z}-k g_{2} f_{x}^{\prime}-g_{3} f_{z}^{\prime \prime}=0
\end{array}
$$

where the constants $g_{1} \ldots g_{4}$ are given by:

$$
\begin{aligned}
g_{1} & =\tilde{\gamma}^{2}\left(9 K \tilde{\gamma}^{8 / 3} \tilde{g}^{2}+5 \tilde{\gamma}^{2} \tilde{g}^{6}+7\right) \\
g_{2} & =\tilde{\gamma}\left(9 K \tilde{\gamma}^{8 / 3} \tilde{g}^{2}-\tilde{g}^{6} \tilde{\gamma}^{2}+4\right) \\
g_{3} & =\left(9 K \tilde{\gamma}^{8 / 3} \tilde{g}^{2}+2 \tilde{\gamma}^{2} \tilde{g}^{6}+10\right) \\
g_{4} & =\frac{g_{2}^{2}}{9 \tilde{\gamma}^{2}}-\frac{g_{1} g_{3}}{9 \tilde{\gamma}^{2}}-9 \tilde{\gamma}^{2} \tilde{g}^{6} \\
& =9 K \tilde{\gamma}^{14 / 3} g^{8}+9 K \tilde{\gamma}^{8 / 3} \tilde{g}^{2}+\tilde{\gamma}^{4} \tilde{g}^{12}+17 \tilde{\gamma}^{2} \tilde{g}^{6}+6
\end{aligned}
$$

As in the incompressible case, uncoupling (79) and (80) yields a constant-coefficient fourth order differential equation in $f_{z}(z)$ :

$$
k^{4} g_{1} f_{z}(z)-k^{2} g_{4} f_{z}^{\prime \prime}(z)+g_{3} \tilde{g}^{6} f_{z}^{(4)}(z)=0
$$

although the coefficients now have algebraically complex dependence on $\gamma$ and $g$, encoded in $g_{1} \ldots g_{4}$.

Solving (79) and (81) gives the form of the displace-
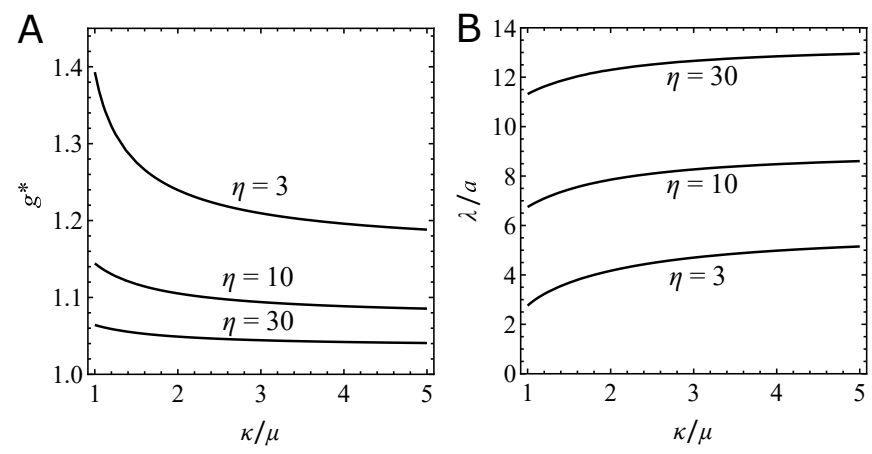

FIG. 10. (A) Threshold growth and (B) wavelength for the first unstable mode for compressible sold with modulus ratio $\eta=3,10$ and 30 as a function of bulk modulus to shear modulus ratio.

ment fields:

$$
f_{z}= \begin{cases}A_{1} e^{k G_{-} z}+A_{2} e^{-k G_{-} z} & +A_{3} e^{k G_{+} z}+A_{4} e^{-k G_{+} z} \\ \left(B_{1}+z B_{2}\right) e^{k z} & z>0, \\ & z<0 .\end{cases}
$$

$f_{x}=\int \frac{9 k^{2} \tilde{\gamma}^{2} f_{z}(z)-g_{3} f_{2}^{\prime \prime}(z)}{g_{2} k} d z$

where two more constants $G_{ \pm}=\sqrt{\frac{g_{4} \pm \sqrt{g_{4}^{2}-4 g_{1} g_{3} g^{6}}}{2 g_{3} g^{6}}}$ have been introduced. Finally, we look at the boundary conditions at the first order. The normal stress boundary condition is, as before,

$$
L_{0}=-\frac{\sigma_{1 z z}}{2 u_{1 z}} .
$$

The rest of the boundary conditions, which are too large to display here, provide linear equations for the constants of integration in the form $M \cdot\left(A_{1}, A_{2}, A_{3}, A_{4}, B_{1}, B_{2}\right)=$ $(\sqrt{2}, 0,0,0,0,0)$, which can be solve to find the form of the constants. As before, the threshold is found by solving (84) with $L_{0}=0$. Again, the solutions for the constants of integration are included the supplementary Mathematica workbook ${ }^{\dagger}$. The result threshold is shown in Fig. 10.

\section{Second order perturbation}

For the next order in $\epsilon$, the same analysis is repeated with higher order fields as with the incompressible case. At second order, the mechanical equilibrium must be satisfied

$$
\nabla \cdot \sigma_{2}=0
$$

At this order, the bulk and the boundary equations become algebraically complicated, making us unable to solve them analytically even with Mathematica. However, the equations are still linear: the complexity arises 

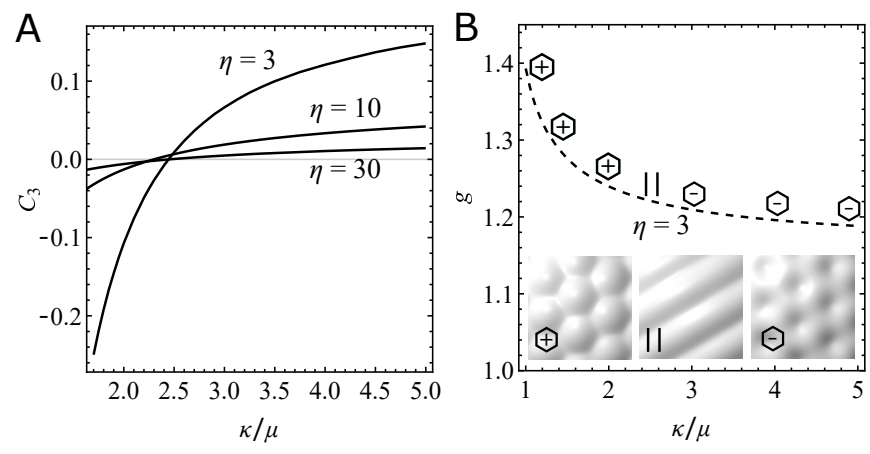

FIG. 11. (A) Coefficient $C_{3}$ as a function of the bulk modulus to shear modulus ratio, $K=\kappa / \mu$ for $\eta=3,10$ and 30. (B) Threshold growth and the patterns closed to the threshold, at $g-g^{*}=2.5 \% \times(g-1)$, as a function of $K$. Images taken from $K=2,2.5$ and 5 respectively.

from the increasing complexity of the surds that form the constant coefficients of the different forms, as we have already seen at first order in $g_{1} \ldots . g_{4}$. Thus, if we insert numerical values for the parameters $(g, k, \eta, K)$, these surds immediately reduce into individual floating point numbers, and Mathematica is then able to solve the system. We thereby solve the equations at second order for $\eta=3$ at the threshold growth $g^{*}$ and use the result to calculate $C_{3}$.

Fig. 11A shows the analytical calculation of coefficient $C_{3}$ as a function of $K$. The key point is that $C_{3}$ changes sign at $K \approx 2.5$ (for $\eta=3$ ) and hence the pattern should change from hexagonal bumps at $K>2.5$ to hexagonal dents at $K<2.5$. This value of bulk modulus to shear modulus ratio is equivalent to a Poisson ratio of $\sim 0.32$, which is a realistic value for many foams, sponges and corks. To confirm this prediction, we conducted many finite element calculations on a large unconstrained domains for systems spanning $K=2.5$. As shown in Fig. 11B the selected pattern changes at $K=2.5$ as expected. Close to $K=2.5$, the stripe pattern appears instead of the hexagon. This is because, as $C_{3}$ approaches zero, the hexagonal pattern loses its advantage of being a subcritical instability. For $\eta=10$ and 30, the sign inversion also appears at slightly different $K$, suggesting that the sign inversion might be generic. Overall, this calculation reveals the importance and value of actually evaluating $C_{3}$ to determine the observed pattern. The calculation at the second order can be found in the supplementary Mathematica workbook ${ }^{\dagger}$, including the solution for given numerical values of parameters, its verification, and computation of $C_{3}$.

\section{IMPOSING INVERSION SYMMETRY}

The non-zero value of $C_{3}$, and the corresponding subcriticality of the hexagonal pattern, has its origin in the lack of inversion symmetry. To emphasize this, we return to the incompressible model and, finally, consider a grow- ing layer sandwiched between an infinitely deep substrate and a matching superstrate. This system has a full inversion symmetry, so $C_{3}$ must vanish, meaning hexagons will no longer be subcritical. In this case, shown in Fig. $12 \mathrm{~A}$, we have:

$$
(\tilde{\mu}, \tilde{g})= \begin{cases}(1,1) & z>a / 2 \\ (\eta, g) & -a / 2<z<a / 2 \\ (1,1) & z<-a / 2\end{cases}
$$

The bulk equations remain the same as in Section V, but the boundary conditions now need to be applied at the top and bottom of the layer, so we now have:

$$
\begin{gathered}
\left.\sigma \cdot \hat{\mathbf{z}}\right|_{z=\frac{a}{2}{ }^{-}}=\left.\sigma \cdot \hat{\mathbf{z}}\right|_{z=\frac{a}{2}+} \\
\left.\sigma \cdot \hat{\mathbf{z}}\right|_{z=-\frac{a}{2}-}=\left.\sigma \cdot \hat{\mathbf{z}}\right|_{z=-\frac{a}{2}+},
\end{gathered}
$$

for the stress boundary conditions, while the displacement continuity conditions become

$$
\begin{aligned}
& \left.\mathbf{u}\right|_{z=\frac{a}{2}{ }^{-}}=\left.\mathbf{u}\right|_{z=\frac{a}{2}+} \\
& \left.\mathbf{u}\right|_{z=\frac{a}{2}-^{-}}=\left.\mathbf{u}\right|_{z=\frac{a}{2}{ }^{+}}
\end{aligned}
$$

Finally, we constrain the amplitude on the upper interface between the growing layer and the superstate, in the same way as before:

$$
E=E_{e l}+\left.L\left\langle u_{z}^{2}-\epsilon^{2}\right\rangle\right|_{z=\frac{a}{2}-} .
$$

The constraint is applied at $z=a / 2^{-}$and thus modifies the stress boundary condition at $z=a / 2$ :

$$
\left.\left(\sigma \cdot \hat{\mathbf{z}}+2 L u_{z} \hat{\mathbf{z}}\right)\right|_{z=\frac{a}{2}-}=\left.\sigma \cdot \hat{\mathbf{z}}\right|_{z=\frac{a}{2}+} .
$$

We can now repeat our analysis up to third order perturbations. At each order, the bulk equations and their solutions have the same form as in the layer/substrate case (as do the energy density expressions $W_{2} \ldots W_{4}$ ), except the substrate solution is now repeated in the superstrate. For example, for stripe patterns, the displacement field at $\mathcal{O}(\epsilon)$ becomes:

$$
f_{z}=\left\{\begin{array}{cc}
e^{-k z}\left(B_{1}+z B_{2}\right) & z>-a / 2, \\
e^{-k z / g^{3}} A_{1}+e^{k z / g^{3}} A_{2}+e^{-k z} A_{3}+e^{k z} A_{4} & -a / 2<z<a / 2, \\
e^{k z}\left(B_{3}+z B_{4}\right) & z<-a / 2 .
\end{array}\right.
$$

However, substituting these fields into the boundary conditions gives different answers for the constants of integration. For example substituting (90) into the boundary conditions give the threshold condition

$$
L_{0}=\left.\frac{\sigma_{1 z z}}{2 u_{1 z}}\right|_{z=\frac{a}{2}+}-\left.\frac{\sigma_{1 z z}}{2 u_{1 z}}\right|_{z=\frac{a}{2}-},
$$

as well as linear equations for the constants of integrations of the form $M\left(A_{1}, A_{2}, A_{3}, A_{4}, B_{1}, B_{2}, B_{3}, B_{4}\right)=$ $(\sqrt{2}, 0,0,0,0,0,0,0)$, where matrix $M$ is given by 


$$
\left(\begin{array}{ccccc}
e^{-\frac{k a}{2 g^{3}}} & e^{\frac{k a}{2 g^{3}}} & e^{-\frac{k a}{2}} & e^{\frac{k a}{2}} & 0 \\
b \eta e^{-\frac{k a}{2 g^{3}}} & b \eta e^{\frac{k a}{2 g^{3}}} & 2 g^{6} \eta e^{-\frac{k a}{2}} & 2 g^{6} \eta e^{\frac{k a}{2}} & -2 g^{2} e^{-\frac{k a}{2}} \\
b \eta e^{\frac{k a}{2 g^{3}}} & b \eta e^{-\frac{k a}{2 g^{3}}} & 2 g^{6} \eta e^{\frac{k a}{2}} & 2 g^{6} \eta e^{-\frac{k a}{2}} & 0 \\
-2 g^{3} \eta e^{\frac{k a}{2 g^{3}}} & 2 g^{3} \eta e^{-\frac{k a}{2 g^{3}}} & -b \eta e^{\frac{k a}{2}} & b \eta e^{-\frac{k a}{2}} & 0 \\
e^{-\frac{k a}{2 g^{3}}} / g^{3} & -e^{\frac{k a}{2 g^{3}}} / g^{3} & e^{-\frac{k a}{2}} & -e^{\frac{k a}{2}} & -e^{-\frac{k a}{2}} \\
e^{-\frac{k a}{2 g^{3}}} & e^{\frac{k a}{2 g^{3}}} & e^{-\frac{k a}{2}} & e^{\frac{k a}{2}} & -e^{-\frac{k a}{2}} \\
e^{\frac{k a}{2 g^{3}}} / g^{3} & -e^{-\frac{k a}{2 g^{3}}} / g^{3} & e^{\frac{k a}{2}} & -e^{-\frac{k a}{2}} & 0 \\
e^{\frac{k a}{2 g^{3}}} & e^{-\frac{k a}{2 g^{3}}} & e^{\frac{k a}{2}} & e^{-\frac{k a}{2}} & 0
\end{array}\right.
$$

$$
\left.\begin{array}{c}
0 \\
0 \\
(a-2 / k) g^{2} e^{-\frac{k a}{2}} \\
a g^{2} e^{-\frac{k a}{2}} \\
0 \\
0 \\
(1 / k-a / 2) e^{-\frac{k a}{2}} \\
a e^{-\frac{k a}{2}} / 2
\end{array}\right)
$$

The threshold is again found by solving $L_{0}=0$, and the resulting threshold and wavelength of the first unstable mode are summarized in Figs 12B and C. By solving for the constants of integration, we can then evaluate $E_{2}$ by integrating $W_{2}$ over the full space. $C_{2}$ can then be obtained as shown in Fig. 12D.

Repeating the analysis at the higher orders, we can determine the coefficients $C_{3}$ and $C_{4}$. As anticipated $C_{3}$ evaluates to zero even for hexagons. $C_{4}$ is plotted as a function of $\eta$ for all patterns in Fig. 12E. In the sandwiched system, squares have the smallest value of $C_{4}$ for $\eta \gtrsim 2$, indicating that squares are the lowest energy pattern close to threshold. However, we note that the differences in $C_{4}$ are rather small, so this conclusion may become inaccurate quite shortly beyond threshold. In the opposite range, $\eta \lesssim 2$, stripes becomes the optimum pattern instead. Finite element analysis on symmetryconstrained systems verifies the amplitude and energy prediction for square patterns and the supercritical nature of the instability (Figs $12 \mathrm{~F}$ and G). Unconstrained finite elements on a large square domain correctly select squares at $\eta=10$ (Fig. 12H). As indicated in Fig. 12B, repeating these simulations at smaller values of $\eta$ confirms the transition to stripe morphologies, in good agreement with the analytic theory. As in previous sections, the full algebraic forms of the solution, verification, and computation the resulting energy coefficients are included in the supplementary Mathematica workbook ${ }^{\dagger}$.

\section{CONCLUSIONS}

Our ambition in this work has been to understand, theoretically, the hexagonal dent patterns that forms close to threshold in the (equibiaxial) compressive buckling of a stiff layer on a soft substrate. Our method essentially mirrors that introduced by Koiter [64] to study the buckling patterns in shells: in short, we use higher order perturbation theory to construct elastic solutions corresponding to patterns with different symmetry, and predict the pattern with the lowest elastic energy. Our approach differs from previous work on layer/substrate buckling, which incorrectly predicts square patterns close to threshold $[42,45]$, because we use a geometrically nonlinear elastic model for both the layer and the substrate, leading to an energy which is not invariant under pattern inversion. Our key finding is that this lack of inversion symmetry underpins the formation of hexagonal dents.

To clarify this link between inversion symmetry and hexagonal pattern, we first minimize the elastic energy over patterns with fixed (rms) amplitude $\epsilon$, and then minimize over $\epsilon$. This allows us, between the two steps, to construct a Landau-like series expansion of the elastic energy in $\epsilon$. In the cases of stripe and square patterns, pattern inversion $(\epsilon \rightarrow-\epsilon)$ simply translates the pattern in the $x-y$ plane, so the energy does not change. This limits the Landau expansion to the form $E=E_{0}+C_{2}\left(g-g^{*}\right) \epsilon^{2}+C_{4} \epsilon^{4}+\mathcal{O}\left(\epsilon^{5}\right)$ and hence produces a supercritical instability. However, for hexagons $\epsilon \rightarrow-\epsilon$ turns a pattern of bumps into a physically different pattern of dents, allowing a $C_{3} \epsilon^{3}$ term in the energy to persist. The hexagonal transition is thus subcritical, strongly suggesting that hexagonal patterns will be favored close to threshold.

This symmetry argument has long been understood in fluids, where it explains the hexagonal patterns formed in Rayleigh-Taylor (gravitational) fingering, Rayleigh Benard convection $[65,66]$, and the Rosensweig ferrofluid instability [67-70]. However, the argument has only recently been applied to solid instabilities, initially by Jia and Ben Amar [53], in the context of the Biot creasing/sulcification instability. In this pioneering paper, the authors considered a soft slab that swells while adhered to a rigid foundation: the $\eta \rightarrow 0$ limit of our system. In their calculation, the elastic fields are truncated at first order, but then the energy is expanded to higher order to evaluate the energy coefficients $\left(C_{2}, C_{3}, C_{4}\right)$ for each pattern. This approach has the correct symmetry, and correspondingly gives a $C_{3}$ term for hexagons. However, since the higher order fields are neglected, the values of $C_{3}$ and $C_{4}$ are only estimates. Indeed, the calculation yields positive $C_{4}$ values for all patterns and hence a prediction of hexagonal patterns, whereas our full high-order calculations reveal that (in the $\eta \rightarrow 0$ limit) $C_{4}$ is negative for all patterns, and hence the optimal pattern cannot be determined. This unusual behavior is a signature of the non-linear onset of the Biot sulcal/crease instability, which puts it beyond the reach of perturbation theory [50], but we note that finite element calculations reveal that square patterns are favored close to threshold [56]. 

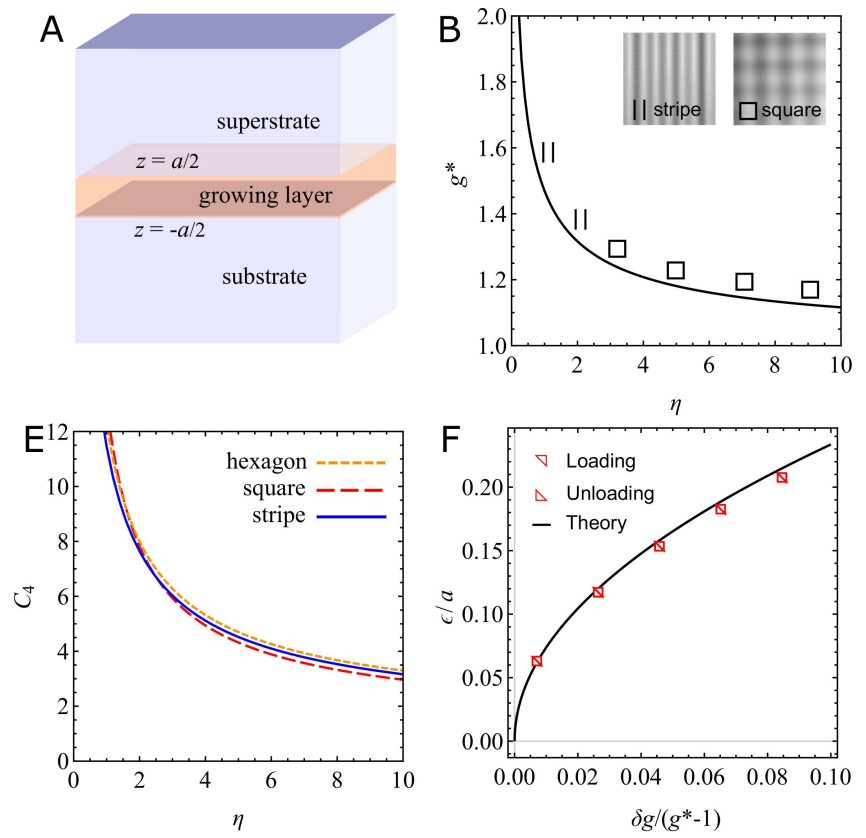
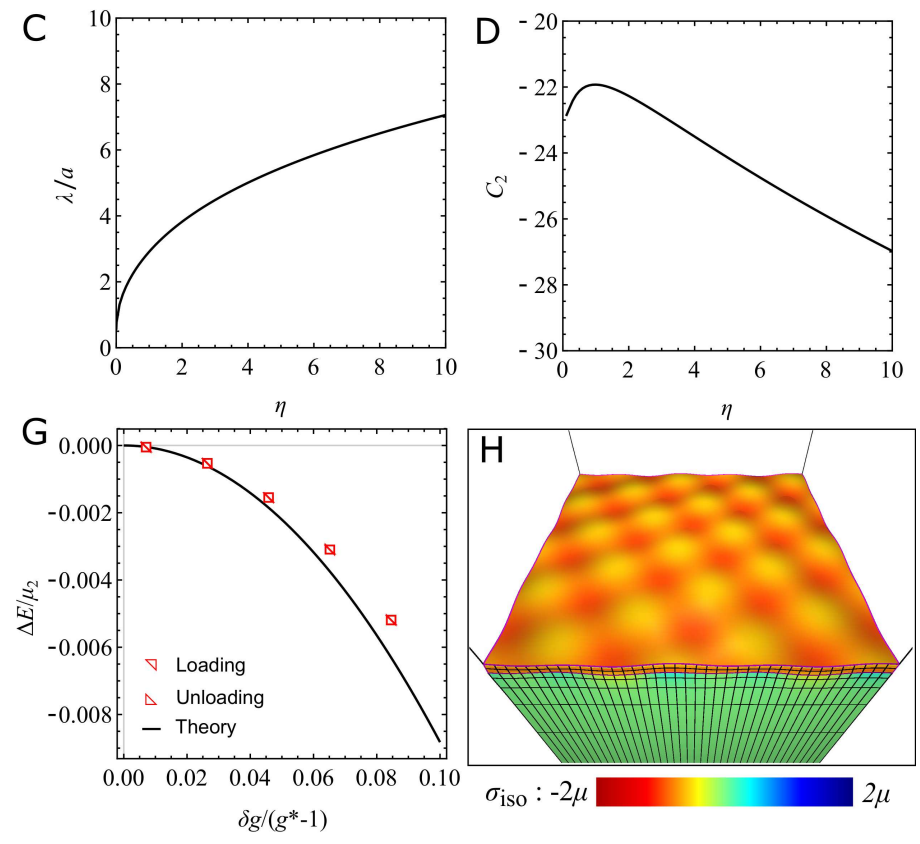

FIG. 12. (A) Schematic diagram for the sandwiched layer system. (B) Threshold growth and the patterns closed to the threshold at $g-g^{*}=5 \% \times\left(g^{*}-1\right)$. (C) Wavelength for the first unstable mode, (D) energy coefficients $C_{2}$ and (E) $C_{4}$ as a function of $\eta$ for a sandwiched layer. (F) Amplitude and $(\mathrm{G})$ energy as a function of growth from the threshold, $\delta g=g-g^{*}$ at $\eta=10$. (H) The square pattern for $\eta=10$ at $g=1.123$.

A year later, Ciarletta [54] conducted a full higher order treatment of the same $\eta \rightarrow 0$ system, but with the addition of surface tension. In the low surface-tension limit, Ciarletta finds negative $C_{4}$ values in agreement with our work, while in the high-surface tension limit $C_{4}$ becomes positive regularizing the instability. However, Ciarletta does not deploy the above symmetry argument at all, because he believes, in our view mistakenly, that the energy has inversion symmetry, and hence that $C_{3}$ is always zero. The resulting expansion is thus unable to distinguish hexagonal dents and bumps, and leads to the conclusion that all patterns are supercritical in the high-surface tension limit (where $C_{4}$ is positive) whereas inclusion of $C_{3}$ would render the hexagonal pattern subcritical.

Finally, very recently, a high-order Koiter method was used by Chakrabarti et al. [71] to analyze pattern formation in solid Rayleigh-Taylor (gravitational) fingering, [72]. These authors conducted a full calculation, and found that $C_{3}>0$, showing that patterns of hexagonal dents will appear subcritically. However, they also find that square patterns are subcritical (i.e,. $C_{4}<0$ ), so the optimal pattern again cannot be formally determined. Fortunately, in this case, finite elements confirm that hexagonal dents are indeed favored.

These previous studies, and our own on layer-substrate buckling, highlight two general messages. The first is the importance of symmetry in the selection of hexagonal patterns: to get the right answer, one must use a theory with the right symmetry. The second is the importance of conducting a full higher order series expansion to actu- ally calculate the higher order energy coefficients, rather than simply relying on the symmetry argument to predict hexagons. The value of $C_{3}$ determines whether one expects up or down hexagons (illustrated by our finite bulk-modulus case) so its value must be explicitly calculated. Furthermore, the value of $C_{4}$ determines whether other patterns are also subcritical, thereby allowing the system to sidestep the symmetry argument altogether. In our case, these full high-order calculations have only been possible via computer algebra, but the good match between our results and our finite element calculations (without any fitting parameters) lends credibility to our results.

In the future, our method could also easily be extended to other elastic systems, including finite depth layer/substrate systems, multi-layer systems, and systems with surface tension. It will also be important to consider the high $\eta$ limit of our fully non-linear theory, to make better contact with the extensive work on plates adhered to linear substrates. Hopefully one can derive a simple effective model for this high stiffness limit, which probably mirrors the previous plate/substrate models, but with some degree of substrate non-linearity. Within such a simplified model, one might succeed in calculating the energy coefficients analytically, rather than resorting to computer algebra. However, as any term that breaks inversion symmetry will lead to hexagonal patterns, there is a real risk of getting the right answer for the wrong reasons, and great care will be needed to identify, self consistently, what non-linear effects should be retained. Within such a model, one might also tackle the 
secondary bifurcation to the ubiquitous herringbone patterns found in stiff/soft systems, and address the width of the post-threshold compression window that gives rise to hexagons.

\section{CONFLICTS OF INTEREST}

There are no conflicts to declare.

\section{APPENDIX}

\section{Appendix A: Details of numerical simulation}

We adapted the code developed by Tuomas Tallinen [47] to use in our problems. The elastic body is broken into tetrahedral elements. In each tetrahedron, the strain and stress are uniform. The stress is calculated on each side of the tetrahedra, using the Cauchy stress for compressible neo-Hookean solid:

$$
T=\mu \frac{(B-I \operatorname{Tr}(B) / 3)}{J^{2 / 3}}+\kappa(J-1) I,
$$

where $B=F \cdot F^{T}$ and $J=\operatorname{Det}(F)$. Except for Section VI, a large bulk modulus, $\kappa=100 \mu$, is used instead of incompressibility to improve the performance of the simulation. Damping was also applied to reduce oscillation in the system. The nodes of the element were displaced using Newton dynamics.

Using finite compressibility would affect our analysis slightly. The threshold value will increase by a small amount from the predicted value (less than $1 \%$ with our values) and the critical wavelength also shifts slightly. Another factor that affects the thresholds are the coarse size of the mesh elements. The size of the elements has to be chosen such that the threshold does not change much from the predicted value but still allowed a sufficient execution speed for the simulation.

Each data point in Figs 5-7,12 are converged close to equilibrium. The simulation were done in a unit cell, with the dimension $L_{x} \times L_{y}$, that can produce each pattern, rectangular cells for the stripe and square, and a $60^{\circ}$-rhomboid cell for the hexagon. The periodic boundary conditions were applied in the $x$ and $y$ directions. The growing layer and the substrate have thickness of $L_{z}$ and $L_{s}$. The corresponding number of mesh elements are given by $n_{x}, n_{y}, n_{z}$ and $n_{s}$. The substrate mesh elements are coarsened in the $z$-direction as they are further away from the growing layer by $19.2 \%$ per element. For determining the appeared pattern, Figs 8C, 11B, 12B and $\mathrm{H}$ were performed a large square domains that can have multiple wavelengths of any patterns. The parameters are summarized in Table I.
TABLE I. Parameters used in finite element analysis

\begin{tabular}{|c|c|c|c|c|}
\hline Fig. & $L_{x} \times L_{y} \times L_{z}$ & $L_{s}$ & $n_{x} \times n_{y} \times n_{z}$ & $n_{s}$ \\
\hline 5 & $5.8 \times 1 \times 1$ & 13.4 & $108 \times 16 \times 26$ & 24 \\
\hline $6 \mathrm{~B}, \mathrm{E}, \mathrm{F}$ & $5.8 \times 5.8 \times 1$ & & & \\
$6 \mathrm{C}, \mathrm{D}$ & $4.4 \times 4.4 \times 1$ & 13.4 & $108 \times 108 \times 26$ & 24 \\
$6 \mathrm{G}, \mathrm{H}$ & $9.1 \times 9.1 \times 1$ & & & \\
\hline 7 & $6.7 \times 6.7 \times 1$ & 13.4 & $108 \times 108 \times 26$ & 24 \\
\hline 8 & $20 \times 20 \times 1$ & 13.4 & $96 \times 96 \times 8$ & 24 \\
\hline 11 & $15 \times 15 \times 1$ & 13.4 & $108 \times 108 \times 26$ & 24 \\
\hline $12 \mathrm{~F}, \mathrm{G}$ & $7.1 \times 7.1 \times 1$ & 13.4 & $60 \times 60 \times 8$ & 24 \\
$12 \mathrm{H}$ & $28 \times 28 \times 1$ & 13.4 & $150 \times 150 \times 8$ & 24 \\
\hline
\end{tabular}

\section{Appendix B: Displacement fields for square and hexagonal patterns}

\section{Square pattern}

The expression for the displacement fields including higher order terms for the square and hexagonal patterns are much more complicated than that of the stripe pattern. For the square pattern, we can write the displacement field as:

$$
\begin{aligned}
u_{z}(x, y, z)= & \epsilon \sum_{n=1}^{2} f_{z, 1, n}(z) \cos \left(\mathbf{k}_{n}^{(1)} \cdot \mathbf{x}\right) \\
& +\epsilon^{2} \sum_{n=1}^{5} f_{z, 2, n}(z) \cos \left(\mathbf{k}_{n}^{(2)} \cdot \mathbf{x}\right) \\
& +\epsilon^{3} \sum_{n=1}^{8} f_{z, 3, n}(z) \cos \left(\mathbf{k}_{n}^{(3)} \cdot \mathbf{x}\right)
\end{aligned}
$$

where $\mathbf{x}=(x, y), \mathbf{k}_{1}^{(1)} \equiv \mathbf{k}_{1}=k \hat{\mathbf{x}}$ and $\mathbf{k}_{2}^{(1)} \equiv \mathbf{k}_{2}=k \hat{\mathbf{y}}$. The notation $\mathbf{k}_{n}^{(i)}$ indicates the $n^{\text {th }}$ component wavevector at the $i^{\text {th }}$ order. Because of the square symmetry, $u_{z}$ must be invariant under rotation symmetry $x \rightarrow y$, $y \rightarrow-x$. There are also reflection symmetries under $x \rightarrow-x$ and under $y \rightarrow-y$. This means we require $f_{z, 1,1}(z)=f_{z, 1,2}(z)$. To construct the ansatz for the next order, we know that the higher order Fourier terms must come from the product of lower order terms. Hence, the possible Fourier components are $\mathbf{k}_{1}^{(2)}=\mathbf{0}, \mathbf{k}_{2}^{(2)}=2 \mathbf{k}_{1}$, $\mathbf{k}_{3}^{(2)}=2 \mathbf{k}_{2}, \mathbf{k}_{4}^{(2)}=\mathbf{k}_{1}+\mathbf{k}_{2}$ and $\mathbf{k}_{5}^{(2)}=\mathbf{k}_{1}-\mathbf{k}_{2}$. Again, symmetry requires $f_{z, 2,2}(z)=f_{z, 2,3}(z)$ and $f_{z, 2,4}(z)=$ $f_{z, 2,5}(z)$. At third order, we need all possible sums of the three instances of $\mathbf{k}_{1}$ or $\mathbf{k}_{2}$, which are

$$
\begin{gathered}
\mathbf{k}_{1}^{(3)}=\mathbf{k}_{1}, \quad \mathbf{k}_{2}^{(3)}=\mathbf{k}_{2}, \quad \mathbf{k}_{3}^{(3)}=3 \mathbf{k}_{1}, \quad \mathbf{k}_{4}^{(3)}=3 \mathbf{k}_{2}, \\
\mathbf{k}_{5}^{(3)}=\mathbf{k}_{1}-2 \mathbf{k}_{2}, \quad \mathbf{k}_{6}^{(3)}=\mathbf{k}_{1}+2 \mathbf{k}_{2}, \\
\mathbf{k}_{7}^{(3)}=2 \mathbf{k}_{1}-\mathbf{k}_{2}, \quad \mathbf{k}_{8}^{(3)}=2 \mathbf{k}_{1}+\mathbf{k}_{2},
\end{gathered}
$$


with $f_{z, 3,1}(z)=f_{z, 3,2}(z), f_{z, 3,3}(z)=f_{z, 3,4}(z)$ and $f_{z, 3,5}(z)=f_{z, 3,6}(z)=f_{z, 3,7}(z)=f_{z, 3,8}(z)$. The form of the field $f_{p, m, n}(z)$ follows from $u_{z}$ with different function $f_{z, m, n}(z)$. The form of $u_{x}$ and $u_{y}$ can be more complicated as they do not have the rotational symmetry but still possess the reflection symmetry (over $x$-axis for $u_{x}$ and $y$-axis for $u_{y}$ ). However, incompressibility condition (9) hints us the form of $u_{x}$ and $u_{z}$ :

$$
\operatorname{Det}(F)-1=\frac{\partial u_{z}}{\partial z}+\frac{\partial u_{x}}{\partial x}+\frac{\partial u_{y}}{\partial y}+O\left(\epsilon^{2}\right)=0
$$

at the lowest order. Their corresponding derivatives must have the Fourier components as that of $u_{z}$ in the same order. We could write down

$$
\begin{aligned}
u_{x}(x, y, z)= & \epsilon \sum_{n=1}^{2} f_{x, 1, n}(z) \sin \left(\mathbf{k}_{n}^{(1)} \cdot \mathbf{x}\right) \\
& +\epsilon^{2} \sum_{n=1}^{5} f_{x, 2, n}(z) \sin \left(\mathbf{k}_{n}^{(2)} \cdot \mathbf{x}\right) \\
& +\epsilon^{3} \sum_{n=1}^{8} f_{x, 3, n}(z) \sin \left(\mathbf{k}_{n}^{(3)} \cdot \mathbf{x}\right) .
\end{aligned}
$$

The function $f_{x, 2,1}(z)=0$ as its term is independent of the $x$ and $y$ coordinate. The $y$-reflection symmetry demands $f_{x, 1,2}(z)=f_{x, 2,3}(z)=f_{x, 3,2}(z)=f_{x, 3,4}(z)=$ 0 , and $f_{x, 2,4}(z)=f_{x, 2,5}(z), f_{x, 3,5}(z)=f_{x, 3,6}(z)$ and $f_{x, 3,7}(z)=f_{x, 3,8}(z)$. Under the rotation transformation $x \rightarrow y, y \rightarrow-x$, the field $u_{x}$ becomes $u_{y}$. Hence, $u_{y}$ can be written as

$$
\begin{aligned}
u_{y}(x, y, z)= & \epsilon \sum_{n=1}^{2} f_{y, 1, n}(z) \sin \left(\mathbf{k}_{n}^{(1)} \cdot \mathbf{x}\right) \\
& +\epsilon^{2} \sum_{n=1}^{5} f_{y, 2, n}(z) \sin \left(\mathbf{k}_{n}^{(2)} \cdot \mathbf{x}\right) \\
& +\epsilon^{3} \sum_{n=1}^{8} f_{y, 3, n}(z) \sin \left(\mathbf{k}_{n}^{(3)} \cdot \mathbf{x}\right),
\end{aligned}
$$

where

$$
\begin{gathered}
f_{y, 1,2}(z)=f_{x, 1,1}, \quad f_{y, 2,3}(z)=f_{x, 2,2}(z), \\
f_{y, 2,4}(z)=-f_{y, 2,5}(z)=f_{x, 2,4}(z), \\
f_{y, 3,2}(z)=f_{x, 3,1}(z), \quad f_{y, 3,4}(z)=f_{x, 3,3}(z), \\
f_{y, 3,5}(z)=-f_{y, 3,6}(z)=-f_{x, 3,7}(z), \\
f_{y, 3,7}(z)=-f_{y, 3,8}=-f_{x, 3,5}(z)
\end{gathered}
$$

while the rest of $f_{y, m, n}(z)$ are zero. With these ansatzes in the displacement fields, we can proceed to solve the Euler-Lagrange equations to obtain the functions $f_{l, m, n}(z)$. The forms of the high-order displacement fields for both the square and hexagonal patterns are included in the supplementary Mathematica workbook. Note that the function $f_{l, m, n}$ are reindexed so that the fields are more compact as there are many zero or duplicated components.

\section{Hexagonal pattern}

For the hexagonal pattern, we start with $u_{z}(x, y, z)=$ $\epsilon \sum_{n=1}^{3} f_{z, 1, n}(z) \cos \left(\mathbf{k}_{n}^{(1)} \cdot \mathbf{x}\right)+O\left(\epsilon^{2}\right)$ where $\mathbf{k}_{1}^{(1)} \equiv \mathbf{k}_{1}=$ $k \hat{\mathbf{x}}, \mathbf{k}_{2}^{(1)} \equiv \mathbf{k}_{2}=k\left(\frac{1}{2}, \frac{\sqrt{3}}{2}\right)=k \hat{\mathbf{x}}_{1}$ and $\mathbf{k}_{3}^{(1)} \equiv \mathbf{k}_{3}=$ $k\left(-\frac{1}{2}, \frac{\sqrt{3}}{2}\right)=k \hat{\mathbf{x}}_{2}$. The hexagonal symmetry requires $u_{z}$ to be invariant under $x \rightarrow x_{1}, x_{1} \rightarrow x_{2}, x_{2} \rightarrow-x$. This means that $f_{z, 1,1}(z)=f_{z, 1,2}(z)=f_{z, 1,3}(z)$. We follow the same principle as before and write the series as in (B1). At second order, the possible wavevectors are:

$$
\begin{gathered}
\mathbf{k}_{1}^{(2)}=\mathbf{0}, \quad \mathbf{k}_{2}^{(2)}=2 \mathbf{k}_{1}, \quad \mathbf{k}_{3}^{(2)}=2 \mathbf{k}_{2}, \quad \mathbf{k}_{4}^{(2)}=2 \mathbf{k}_{3}, \\
\mathbf{k}_{5}^{(2)}=\mathbf{k}_{2}-\mathbf{k}_{3}=\mathbf{k}_{1}, \quad \mathbf{k}_{6}^{(2)}=\mathbf{k}_{3}+\mathbf{k}_{1}=\mathbf{k}_{2}, \\
\mathbf{k}_{7}^{(2)}=\mathbf{k}_{2}-\mathbf{k}_{1}=\mathbf{k}_{3}, \quad \mathbf{k}_{8}^{(2)}=\mathbf{k}_{1}+\mathbf{k}_{2} \\
\mathbf{k}_{9}^{(2)}=\mathbf{k}_{2}+\mathbf{k}_{3}, \quad \mathbf{k}_{10}^{(2)}=\mathbf{k}_{1}-\mathbf{k}_{3} .
\end{gathered}
$$

From symmetry, the relations between the function $f_{z, 2, n}(z)$ are

$$
\begin{aligned}
& f_{z, 2,2}(z)=f_{z, 2,3}(z)=f_{z, 2,4}(z) \\
& f_{z, 2,5}(z)=f_{z, 2,6}(z)=f_{z, 2,7}(z), \\
& f_{z, 2,8}(z)=f_{z, 2,9}(z)=f_{z, 2,10}(z) .
\end{aligned}
$$

At third order, we have

$$
\begin{gathered}
\mathbf{k}_{1}^{(3)}=\mathbf{k}_{1}-\mathbf{k}_{2}+\mathbf{k}_{3}=\mathbf{0}, \\
\mathbf{k}_{2}^{(3)}=\mathbf{k}_{1}, \quad \mathbf{k}_{3}^{(3)}=\mathbf{k}_{2}, \quad \mathbf{k}_{4}^{(3)}=\mathbf{k}_{3}, \\
\mathbf{k}_{5}^{(3)}=2 \mathbf{k}_{1}, \quad \mathbf{k}_{6}^{(3)}=2 \mathbf{k}_{2}, \quad \mathbf{k}_{7}^{(3)}=2 \mathbf{k}_{3}, \\
\mathbf{k}_{8}^{(3)}=3 \mathbf{k}_{1}, \quad \mathbf{k}_{9}^{(3)}=3 \mathbf{k}_{2}, \quad \mathbf{k}_{10}^{(3)}=3 \mathbf{k}_{3}, \\
\mathbf{k}_{11}^{(3)}=\mathbf{k}_{1}+\mathbf{k}_{2}, \quad \mathbf{k}_{12}^{(3)}=\mathbf{k}_{2}+\mathbf{k}_{3}, \quad \mathbf{k}_{13}^{(3)}=\mathbf{k}_{1}-\mathbf{k}_{3}, \\
\mathbf{k}_{14}^{(3)}=2 \mathbf{k}_{1}+\mathbf{k}_{2}, \quad \mathbf{k}_{15}^{(3)}=2 \mathbf{k}_{2}+\mathbf{k}_{3}, \quad \mathbf{k}_{16}^{(3)}=2 \mathbf{k}_{3}-\mathbf{k}_{1},
\end{gathered}
$$


$\mathbf{k}_{17}^{(3)}=2 \mathbf{k}_{1}-\mathbf{k}_{3}, \quad \mathbf{k}_{18}^{(3)}=2 \mathbf{k}_{2}+\mathbf{k}_{1}, \quad \mathbf{k}_{19}^{(3)}=2 \mathbf{k}_{3}+\mathbf{k}_{2}$,

and the rotation and reflection symmetries require

$$
\begin{gathered}
f_{z, 3,2}(z)=f_{z, 3,3}(z)=f_{z, 3,4}(z) \\
f_{z, 3,5}(z)=f_{z, 3,6}(z)=f_{z, 3,7}(z) \\
f_{z, 3,8}(z)=f_{z, 3,9}(z)=f_{z, 3,10}(z) \\
f_{z, 3,11}(z)=f_{z, 3,12}(z)=f_{z, 3,13}(z) \\
f_{z, 3,14}(z)=f_{z, 3,15}(z)=f_{z, 3,16}(z)=f_{z, 3,17}(z) \\
=f_{z, 3,18}(z)=f_{z, 3,19}(z) .
\end{gathered}
$$

The full form of $u_{z}$ is hence

$$
\begin{aligned}
u_{z}(x, y, z)= & \epsilon \sum_{n=1}^{3} f_{z, 1, n}(z) \cos \left(\mathbf{k}_{n}^{(1)} \cdot \mathbf{x}\right) \\
& +\epsilon^{2} \sum_{n=1}^{10} f_{z, 2, n}(z) \cos \left(\mathbf{k}_{n}^{(2)} \cdot \mathbf{x}\right) \\
& +\epsilon^{3} \sum_{n=1}^{19} f_{z, 3, n}(z) \cos \left(\mathbf{k}_{n}^{(3)} \cdot \mathbf{x}\right) .
\end{aligned}
$$

The displacement field $u_{x}$ and $u_{y}$ are more difficult to construct than the square pattern case as there is no $x-y$ symmetry. However, the general form similar to (B3) and (B4) can still be used:

$$
\begin{aligned}
u_{x}(x, y, z)= & \epsilon \sum_{n=1}^{3} f_{x, 1, n}(z) \sin \left(\mathbf{k}_{n}^{(1)} \cdot \mathbf{x}\right) \\
& +\epsilon^{2} \sum_{n=1}^{10} f_{x, 2, n}(z) \sin \left(\mathbf{k}_{n}^{(2)} \cdot \mathbf{x}\right) \\
& +\epsilon^{3} \sum_{n=1}^{19} f_{x, 3, n}(z) \sin \left(\mathbf{k}_{n}^{(3)} \cdot \mathbf{x}\right), \\
u_{y}(x, y, z)= & \epsilon \sum_{n=1}^{3} f_{y, 1, n}(z) \sin \left(\mathbf{k}_{n}^{(1)} \cdot \mathbf{x}\right) \\
& +\epsilon^{2} \sum_{n=1}^{10} f_{y, 2, n}(z) \sin \left(\mathbf{k}_{n}^{(2)} \cdot \mathbf{x}\right) \\
& +\epsilon^{3} \sum_{n=1}^{19} f_{y, 3, n}(z) \sin \left(\mathbf{k}_{n}^{(3)} \cdot \mathbf{x}\right) .
\end{aligned}
$$

We cannot have $x$ or $y$ independent components for $u_{x}$ and $u_{y}$ so $f_{x, 2,1}(z)=f_{x, 3,1}(z)=f_{y, 2,1}(z)=f_{y, 3,1}(z)=$ 0 . The $x$-axis reflection symmetry grants the following relations for $u_{x}$ :

$$
f_{x, 1,2}(z)=-f_{x, 1,3}(z), \quad f_{x, 2,3}(z)=-f_{x, 2,4}(z),
$$

$$
f_{x, 2,6}(z)=-f_{x, 2,7}(z), \quad f_{x, 2,8}(z)=f_{x, 2,10}(z)
$$

$$
f_{x, 2,9}(z)=0, \quad f_{x, 3,3}(z)=-f_{x, 3,4}(z)
$$

$$
f_{x, 3,6}(z)=-f_{x, 3,7}(z), \quad f_{x, 3,9}(z)=-f_{x, 3,10}(z),
$$

$$
f_{x, 3,11}(z)=f_{x, 3,13}(z), \quad f_{x, 3,12}(z)=0
$$

$$
f_{x, 3,14}(z)=f_{x, 3,16}(z), \quad f_{x, 3,15}(z)=-f_{x, 3,19}(z),
$$

$$
f_{x, 3,16}(z)=-f_{x, 3,18}(z),
$$

and the $y$-axis reflection symmetry grants the following relations for $u_{y}$ :

$$
\begin{gathered}
f_{y, 1,1}(z)=f_{y, 2,2}(z)=f_{y, 2,5}(z)=f_{y, 3,2}(z) \\
=f_{y, 3,5}(z)=f_{y, 3,8}(z)=0, \\
f_{y, 1,2}(z)=f_{y, 1,3}(z), \quad f_{y, 2,3}(z)=f_{y, 2,4}(z), \\
f_{y, 2,6}(z)=f_{y, 2,7}(z), \quad f_{y, 2,8}(z)=-f_{y, 2,10}(z), \\
f_{y, 3,3}(z)=f_{y, 3,4}(z), \quad f_{y, 3,6}(z)=f_{y, 3,7}(z), \\
f_{y, 3,9}(z)=f_{y, 3,10}(z), \quad f_{y, 3,11}(z)=-f_{y, 3,13}(z), \\
f_{y, 3,14}(z)=-f_{y, 3,17}(z), \quad f_{y, 3,15}(z)=f_{y, 3,19}(z), \\
f_{y, 3,16}(z)=f_{y, 3,18}(z) .
\end{gathered}
$$

To find relations between these functions, we can use the rotational symmetry of the displacement field $\mathbf{u}=$ $\left(u_{x}, u_{y}, u_{z}\right)$. The field is invariant under A 60-degree rotation around $\hat{\mathbf{z}}$ with $x \rightarrow x_{1}, x_{1} \rightarrow x_{2}, x_{2} \rightarrow-x$ transformation. At first order, working in $x-y$ basis, we must have

$$
\begin{gathered}
\left(\begin{array}{cc}
1 / 2 & -\sqrt{3} / 2 \\
\sqrt{3} / 2 & 1 / 2
\end{array}\right)\left(\begin{array}{c}
f_{x, 1,1}(z) \sin \left(k x_{1}\right)+ \\
f_{x, 1,2}(z)\left(\sin \left(k x_{2}\right)+\sin (k x)\right) \\
f_{y, 1,2}(z)\left(\sin \left(k x_{2}\right)-\sin (k x)\right)
\end{array}\right) \\
=\left(\begin{array}{c}
f_{x, 1,1}(z) \sin (k x)+ \\
f_{x, 1,2}\left(\sin \left(k x_{1}\right)-\sin \left(k x_{2}\right)\right) \\
f_{y, 1,2}(z)\left(\sin \left(k x_{1}\right)+\sin (k x 2)\right)
\end{array}\right) .
\end{gathered}
$$

Solving the equations give us

$$
f_{x, 1,1}(z)=2 f_{x, 1,2}(z)=\frac{2 f_{y, 1,2}(z)}{\sqrt{3}} .
$$


Using this principle for the higher order components, we obtain:

$$
\begin{gathered}
f_{x, 2,2}(z)=2 f_{x, 2,3}(z)=\frac{2 f_{y, 2,3}(z)}{\sqrt{3}}, \\
f_{x, 2,5}(z)=2 f_{x, 2,6}(z)=\frac{2 f_{y, 2,6}(z)}{\sqrt{3}}, \\
f_{x, 2,8}(z)=\sqrt{3} f_{y, 2,8}(z)=\frac{\sqrt{3} f_{y, 2,9}(z)}{2}, \\
f_{x, 3,2}(z)=2 f_{x, 3,3}(z)=\frac{2 f_{y, 3,3}(z)}{\sqrt{3}},
\end{gathered}
$$

$$
\begin{gathered}
f_{x, 3,5}(z)=2 f_{x, 3,6}(z)=\frac{2 f_{y, 3,6}(z)}{\sqrt{3}}, \\
f_{x, 3,8}(z)=2 f_{x, 3,9}(z)=\frac{2 f_{y, 3,9}(z)}{\sqrt{3}}, \\
f_{x, 3,11}(z)=\sqrt{3} f_{y, 3,11}(z)=\frac{\sqrt{3} f_{y, 3,12}(z)}{2} .
\end{gathered}
$$

\section{ACKNOWLEDGEMENTS}

We thank T. Tallinen, on whose code our finite element calculations are based. N.C. thanks the Royal Thai Government Scholarship for funding.
[1] H. G. Allen, Analysis and Design of Structural Sandwich Panels: The Commonwealth and International Library: Structures and Solid Body Mechanics Division (Pergamon Press, 1969).

[2] J. Dervaux, Y. Couder, M.-A. Guedeau-Boudeville, and M. B. Amar, Phys. Rev. Lett. 107, 018103 (2011).

[3] P. Ciarletta and M. B. Amar, Int. J. Non Linear Mech. 47, 248 (2012).

[4] M. Kücken and A. Newell, EPL (Europhysics Letters) 68, 141 (2004).

[5] M. Diab, T. Zhang, R. Zhao, H. Gao, and K.-S. Kim, Proc. R. Soc. A 469, 20120753 (2013).

[6] M. J. Razavi, R. Pidaparti, and X. Wang, Phys. Rev. E 94, 022405 (2016).

[7] D. E. Moulton and A. Goriely, J. Mech. Phys. Solids 59, 527 (2011).

[8] B. Li, Y.-P. Cao, X.-Q. Feng, and H. Gao, J. Mech. Phys. Solids 59, 758 (2011).

[9] K. Efimenko, M. Rackaitis, E. Manias, A. Vazili, L. Mahadevan, and J. Genzer, Nat. Mater. 4, 293 (2005).

[10] D. P. Richman, R. M. Steward, J. W. Hutchinson, and V. S. J. Caviness, Science 189, 18 (1975).

[11] G. Xu, A. K. Knudsen, K. Dikranian, C. D. Kroenke, P. V. Bayly, and L. A. Taber, J. Biomech. Eng. 132, 071013 (2010).

[12] R. Toro and Y. Burnod, Cereb. Cortex 15, 1900 (2005).

[13] P. V. Bayly, R. J. Okamoto, G. Xu, Y. Shi, and L. A. Taber, Phys. Biol. 10, 016005 (2013).

[14] S. Budday, P. Steinmann, and E. Kuhl, J. Mech. Phys. Solids 72, 75 (2014).

[15] T. Tallinen, J. Y. Chung, J. S. Biggins, and L. Mahadevan, PNAS 111, 12667 (2014).

[16] T. Tallinen, J. Y. Chung, F. Rosseau, N. Girard, J. Lefevre, and L. Mahadevan, Nat. Phys. 12, 588 (2016).

[17] E. Hannezo, J. Prost, and J. F. Joanny, Phys. Rev. Lett. 107, 078104 (2011).

[18] T. Savin, N. A. Kurpios, A. E. Shyer, P. Florescu, H. Liang, L. Mahadevan, and C. J. Tabin, Nature 476, 57 (2011).

[19] A. E. Shyer, T. Tallinen, N. L. Nerurkar, Z. Wei, E. S. Gil, D. L. Kaplan., C. J. Tabin, and L. Mahadevan,
Science 342, 212 (2013).

[20] J. Marthelot, P.-T. Brun, F. L. Jiménez, , and P. M. Reis, Phys. Rev. Materials 1, 025601 (2017).

[21] D. Wang, N. Cheewaruangroj, Y. Li, G. McHale, Y. Jiang, D. Wood, J. S. Biggins, , and B. B. Xu, Adv. Funct. Mater. 28, 1704228 (2018).

[22] P.-C. Lin, S. Vajpayee, A. Jagota, C.-Y. Huid, and S. Yang, Soft Matter 4, 1830 (2008).

[23] E. P. Chan, E. J. Smith, R. C. Hayward, and A. J. Crosby, Adv. Mater. 20, 711 (2008).

[24] K. Khare, J. Zhou, and S. Yang, Langmuir 25, 12794 (2009).

[25] S. G. Lee, D. Y. Lee, H. S. Lim, D. H. Lee, S. Lee, and K. Cho, Adv. Mater. 22, 5013 (2010).

[26] X. Huang., Y. Sun, and S. Soh, Adv. Mater. 27, 4062 (2015).

[27] P. Görrn, M. Lehnhardt, W. Kowalsky, T. Riedl, and S. Wagner, Adv. Mater. 23, 869 (2011).

[28] E. Lee, M. Zhang, Y. Cho, Y. Cui, J. V. der Spiegel, N. Engheta, and S. Yang, Adv. Mater. 26, 4127 (2014).

[29] S. Zeng, D. Zhang, W. Huang, Z. Wang, S. G. Freire, X. Yu, A. T. Smith, E. Y. Huang, H. Nguon, and L. Sun, Nat. Commun. 7, 11802 (2016).

[30] J. Shim, C. Perdigou, E. R. Chen, K. Bertoldi, and P. M. Reis, PNAS 109, 5978 (2012).

[31] P. Christian, H. M. A. Ehmann, O. Werzerbc, and A. M. Coclite, Soft Matter 12, 9501 (2016).

[32] R. Huang and Z. Suo, Int. J. Solids Struct. 39, 1791 (2002).

[33] Z. Y. Huang, W. Hong, and Z. Suo, J. Mech. Phys. Solids 53, 2101 (2005).

[34] H. Jiang, D.-Y. Khang, J. Song, Y. Sun, Y. Huang, and J. A. Rogers, PNAS 104, 15607 (2007).

[35] H. Jiang, D.-Y. Khang, H. Fei, H. Kim, Y. Huang, J. Xiao, and J. A. Rogers, J. Mech. Phys. Solids 56, 2595 (2008).

[36] S. Wang, J. Song, D.-H. Kim, Y. Huang, and J. A. Rogers, Appl. Phys. Lett. 93, 023126 (2008).

[37] J. Song, H. Jiang, Z. Liu, D. Khang, Y. Huang, J. A. Rogers, C. Luc, and C. Koh, Int. J. Solids Struct. 45, 3107 (2008). 
[38] S. H. Im and R. Huang, J. Mech. Phys. Solids 56, 3315 (2008).

[39] Y. Cao and J. W. Hutchinson, J. Appl. Mech. 79, 031019 (2012).

[40] D. Breid and A. J. Crosby, Soft Matter 5, 425 (2009).

[41] M. Guvendiren, J. A. Burdick, and S. Yang, Soft Matter 6, 5795 (2010).

[42] S. Cai, D. Breid, A. J. Crosby, Z. Suo, and J. W. Hutchinson, J. Mech. Phys. Solids 59, 1094 (2011).

[43] E. Cerda and L. Mahadevan, Phys. Rev. Lett. 90, 074302 (2003).

[44] X. Chen and J. W. Hutchinson, J. Appl. Mech. 71, 597 (2004).

[45] B. Audoly and A. Boudaoud, Phys. Solids 56, 2401 (2008).

[46] D. Breid and A. J. Crosby, Soft Matter 7, 4490 (2011).

[47] T. Tallinen and J. S. Biggins, Phys. Rev. E 92, 022720 (2015).

[48] M. A. Biot, Appl. Sci. Res. 12, 168 (1963).

[49] M. A. Biot, Mechanics of Incremental Deformations (Wiley, New York, 1965).

[50] E. Hohlfeld and L. Mahadevan, Phys. Rev. Lett. 106, 105702 (2011).

[51] S. Cai, D. Chen, Z. Suo, and R. C. Hayward, Soft Matter 8, 1301 (2012).

[52] M. B. Amar and P. Ciarletta, J. Mech. Phys. Solids 58, 935 (2010).

[53] F. Jia and M. B. Amar, Soft Matter 9, 8216 (2013).

[54] P. Ciarletta, Soft Matter 9, 8216 (2013).

[55] V. Trujillo, J. Kim, and R. C. Hayward, Soft Matter 4, 564 (2008).

[56] T. Tallinen, J. S. Biggins, and L. Mahadevan, Phys. Rev. Lett. 110, 024302 (2013).

[57] S. Budday, S. Andres, B. Walter, P. Steinmann, and E. Kuhl, Phil. Trans. R. Soc. A 375, 20160163 (2016).
[58] M. A. Holland, B. Li, X. Feng, and E. Kuhl, J. Mech. Phys. Solids 98, 350 (2017).

[59] A. Auguste, L. Jin, Z. Suo, and R. C. Hayward, Extreme Mechanics Letters 11, 30 (2017).

[60] Y. Cao and J. W. Hutchinson, Proc. R. Soc. A 468, 94 (2012).

[61] J. W. Hutchinson, Phil. Trans. R. Soc. 371, 20120422 (2013).

[62] L. Jin, A. Takei, and J. W. Hutchinson, J. Mech. Phys. Solids 81, 22 (2015).

[63] Y. Fu and G. A. Rogerson, Proc. R. Soc. Lond. A 446, 233 (1994).

[64] W. T. Koiter, Over de stabiliteit van het elastisch evenwicht (On the Stability of Elastic Equilibrium), Ph.D. thesis, Polytechnic Institute of Delft (1945).

[65] H. Bénard, Annales de chimie et de physique (1901).

[66] L. R. O. F.R.S., The London, Edinburgh, and Dublin Philosophical Magazine and Journal of Science 32, 529 (1916).

[67] M. D. Cowley and R. E. Rosenweig, J. Fluid Mech. 30, 671 (1967).

[68] A. Gailitis, J. Fluid Mech. 82, 401 (1977).

[69] Y. Cao and Z. J. Ding, J. Magn. Magn. Mater. 355, 93 (2014).

[70] C. Gollwitzer, I. Rehberg, and R. Richter, J. Phys.: Condens. Matter 18, S2643 (2006).

[71] A. Chakrabarti, S. Mora, F. Richard, T. Phou, J.-M. Fromental, Y. Pomeau, and B. Audoly, J. Mech. Phys. Solids 121, 234 (2018).

[72] S. Mora, T. Phou, J.-M. Fromental, and Y. Pomeau, Phys. Rev. Lett. 113, 178301 (2014). 